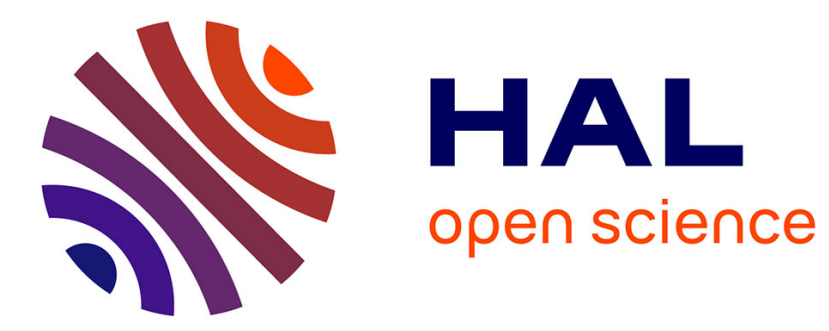

\title{
Reasoning with a Network of Aligned Ontologies
}

Antoine Zimmermann, Chan Le Duc

\section{To cite this version:}

Antoine Zimmermann, Chan Le Duc. Reasoning with a Network of Aligned Ontologies. [Research Report] RR-6484, INRIA. 2008, pp.41. inria-00267808v3

\section{HAL Id: inria-00267808 https://hal.inria.fr/inria-00267808v3}

Submitted on 27 Jun 2008

HAL is a multi-disciplinary open access archive for the deposit and dissemination of scientific research documents, whether they are published or not. The documents may come from teaching and research institutions in France or abroad, or from public or private research centers.
L'archive ouverte pluridisciplinaire HAL, est destinée au dépôt et à la diffusion de documents scientifiques de niveau recherche, publiés ou non, émanant des établissements d'enseignement et de recherche français ou étrangers, des laboratoires publics ou privés. 


\title{
I N R I A
}

INSTITUT NATIONAL DE RECHERCHE EN INFORMATIQUE ET EN AUTOMATIQUE

\section{Reasoning with a Network of Aligned Ontologies}

\author{
Antoine Zimmermann — Chan Le Duc
}

$\mathrm{N}^{\circ} 6484$

March 2008

Thème SYM

apport

de recherche 



\title{
RIN RIA
}

\section{Reasoning with a Network of Aligned Ontologies}

\author{
Antoine Zimmermann, Chan Le Duc \\ Thème SYM — Systèmes symboliques \\ Projet EXMO \\ Rapport de recherche $\mathrm{n}^{\circ} 6484$ - March 2008 - 38 pages
}

\begin{abstract}
In the context of the Semantic Web or semantic P2P systems, many ontologies may exist and be developed independently. Ontology alignments help integrating, mediating or simply reasoning with a system of networked ontologies. Though different formalisms have already been defined to reason with such systems, they do not consider ontology alignments as first class objects designed by third party ontology matching systems. Correspondences between ontologies are often asserted from an external point of view encompassing both ontologies. We propose a formalism, Integrated Distributed Description Logics (IDDL), which treats local knowledge (ontologies) and global knowledge (inter-ontology semantic relations, i.e., alignments) separately by distinguishing local interpretations and global interpretation. In this report, we identify relevant requirements for the semantics of such distributed systems. From this analysis, we argue that IDDL complies with these requirements. We then present a reasoning procedure for IDDL systems which uses local reasoners in a modular way. It proves that consistency of a IDDL system is decidable iff consistency of the local logics is decidable and it provides an upper bound for the complexity of consistency checking.
\end{abstract}

Key-words: Distributed Knowledge, Ontology Alignment, Description Logics. 


\section{Raisonnement sur un réseau d'ontologies alignées Rapport de recherche INRIA}

Résumé : Dans le contexte du Web sémantique ou des réseaux pair-à-pair sémantique, de nombreuses ontologies peuvent être développées indépendemment. Les alignements d'ontologies aident à l'intégration, la médiation ou au raisonnement dans un système d'ontologies connectées. Bien que différents formalismes existent déjà pour raisonner sur de tels systèmes, ils ne considèrent pas les alignements d'ontologies comme des objets de première classe, conçus par des systèmes de mise en correspondance tiers partie. Les correspondances entre ontologies sont souvent énoncées d'un point de vue externe englobant les deux ontologies. Nous proposons un formalisme, Integrated Distributed Description Logics (IDDL), qui traite les connaissances locales (les ontologies) et les connaissances globales (relations sémantiques inter-ontologie, c'est-à-dire les alignments) séparément en distinguant les interpretations locales et l'interpretation globale. Dans ce rapport, nous identifions des critères pertinents pour la sémantique de tels systèmes distribués. De cette analyse, nous soutenons que IDDL est conforme à ces critères. Nous présentons ensuite une procédure de raisonnement pour des systèmes distribués IDDL qui utilise des raisonneurs locaux de façon modulaire. Cela prouve que la consistence d'un système IDDL est décidable si les logiques locales sont décidables et cela fournit une borne supérieure pour la complexité de la vérification de consistence.

Mots-clés : connaissances distribuées, alignement d'ontologies, logiques de description. 


\section{Contents}

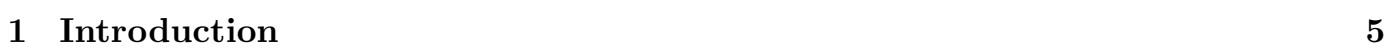

2 Motivating Example and Requirements $\quad 6$

2.1 Motivating Example . . . . . . . . . . . . . . . . . . . . . 6

2.2 Requirements ........................... 7

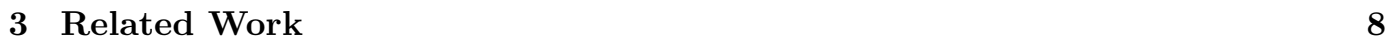

$3.1 \quad$ Merging-based approach $\ldots \ldots \ldots \ldots \ldots$



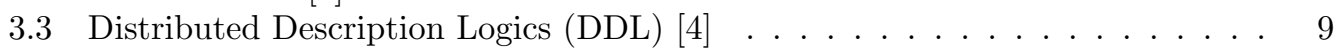

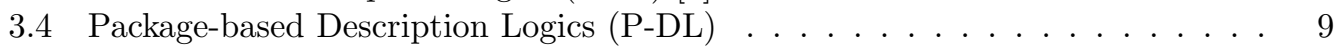

$4 \quad$ Integrated Distributed Description Logics (IDDL) 9

4.1 DL: syntax and semantics . . . . . . . . . . . . . . . . . . . 10

4.2 Distributed Systems . . . . . . . . . . . . . . . . . . . . . . 10

4.2 .1 Syntax: . . . . . . . . . . . . . . . . . . . . . . . . . . . . . . . . . . . . . . .

4.2 .2 Semantics . . . . . . . . . . . . . . . . . . . . . . 11



5.1 Configurations and extended ontologies . . . . . . . . . . . . . . . 14

$\begin{array}{lll}6 & \text { Theorem and proof } & 16\end{array}$

6.1 If-direction $€ \ldots \ldots \ldots \ldots \ldots$

6.1 .1 Building the equalizing function. . . . . . . . . . . . . . . 17

6.1 .2 Auxiliary lemma. . . . . . . . . . . . . . . . . . . . . . . . . . . . . . . . . 18

6.1.3 Satisfaction of the system. . . . . . . . . . . . . . . . . . . . . . . . . . . . . . . .

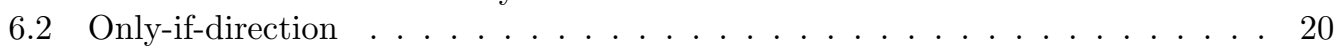

6.2 .1 Building configurations. . . . . . . . . . . . . . . . . . . . . . . . . . . . . . . . . . . . .

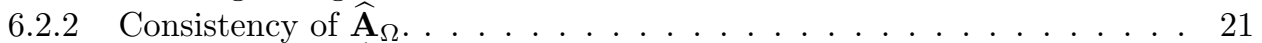

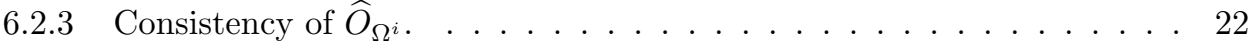

\begin{tabular}{|lll}
\hline 7 & An Algorithm for Consistency Checking & 24
\end{tabular}

8 Reasoning with Cross-ontology Role Subsumption 27

8.1 If-direction $€ \ldots \ldots \ldots \ldots \ldots \ldots$

8.1 .1 Auxiliary lemmas. . . . . . . . . . . . . . . . . . . . . . . 29

8.1 .2 Satisfaction of the system. . . . . . . . . . . . . . . . . . . . . . . . . . . . . .

8.2 Only-if-direction $\ldots \ldots \ldots \ldots \ldots$

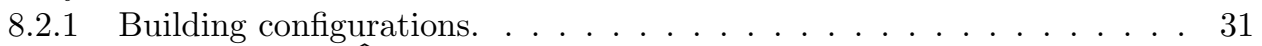

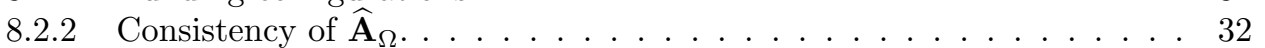

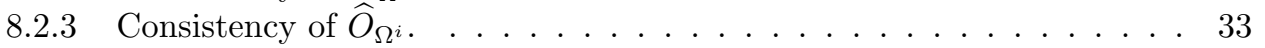


9 Conclusion and Future Work 


\section{Introduction}

The World Wide Web became more and more popular by providing tools that enables users to easily publish web pages. Similarly, the Semantic Web will offer tools to semantically annotate documents, or create ontologies. Therefore, light-weight personal ontologies will most likely cohabit with large institutional ontologies. Reasoning with multiple ontologies that are developed independently is critical to the viability of the Semantic Web. For that matter, ontology matching techniques are used. However, ontology matchers usually do not define a formal semantic for the alignments they produce. Besides, several formalisms were introduced in order to reason with a network of ontologies connected with alignments. Nonetheless, we pretend that they do not properly comply with the common situation where ontologies are developed independently and the alignments are computed separately.

In this paper, we define a language which serves to describe networked ontologies and we provide its formal semantics. We argue that its design comply with the aforementioned notions. More precisely, the language distinguishes reasoning with locally defined ontologies (in Description Logics) and reasoning with the alignments and ontologies together (global reasoning). Each local ontology can be interpreted in its own domain of interpretation, while the whole ontology network is interpreted in a unique global domain of interpretation. Local domains are correlated into the global domain through the use of "equalizing function" that associates elements in the local domain to elements in the global domain. Most importantly, we present an algorithm for reasoning with such formalism. The existence of this algorithm proves the decidability of our formalism in function of the decidability of local ontology languages. Moreover, checking consistency of an ontology network is made by taking advantage of local reasoners, so that the global reasoner does not need to actually access the content of local ontologies. Furthermore, the algorithm can check the consistency of a system regardless of the expressiveness of local logics.

In Sect. 2, we start by giving a motivating examples that helps identifying our requirements. In Sect. 3, we present existing reasoning formalisms and explain how they fail in the context we are interested in. Sect. 4 presents the formalism itself: Integrated Distributed Description Logics (IDDL) - Syntax and Semantics. The main contribution is a reasoning procedure which determines whether or not a IDDL distributed system is consistent. Reasoning in IDDL imposes the construction of several structures which are difficult to introduce in a short space. The definitions of these structures impose quite heavy notations. Therefore, for readability reason, we first consider the case when only concepts are put in correspondences of alignments. First, Sect. 5 contains the formal definitions with textual explanations that will guide the reader in understanding our procedure (and will ease the understanding of the more general case including role correspondences). Second, Sect. 6 presents a theorem (Theo. 1) which gives a criteria for determining consistency of a distributed system and the (long) proof follows. Third, an algorithm is proposed in Sect. 7. We consider role correspondences in Sect. 8, which adds new definitions, proofs and modifications to the algorithm. Sect. 9 adds concluded remarks, a small discussion and further work. 


\section{Motivating Example and Requirements}

Some particularities of our proposed formalism were already promoted in [22, [21] and [9]. This section improves the considerations found in these articles by providing a concrete motivating example (Sect. 2.1) that leads to a more detailed presentation of the requirements that support the specificities of IDDL (Sect. 2.2.

\section{$2.1 \quad$ Motivating Example}

In the following example, we consider a network of 3 independently developed ontologies $O_{1}$, $\mathrm{O}_{2}$ and $\mathrm{O}_{3}$ connected via alignments $A_{12}, A_{13}$ and $A_{23}$ probably computed by third party ontology matching tools. Although these ontologies are very small, one can imagine that they represent the relevant part of bigger real life ontologies. The first ontology is about geopolitical information, including rulers, countries, etc. The second is an ontology of the biological kingdom Animalia. The third one is about geographical regions on Earth (see Fig 2.1.

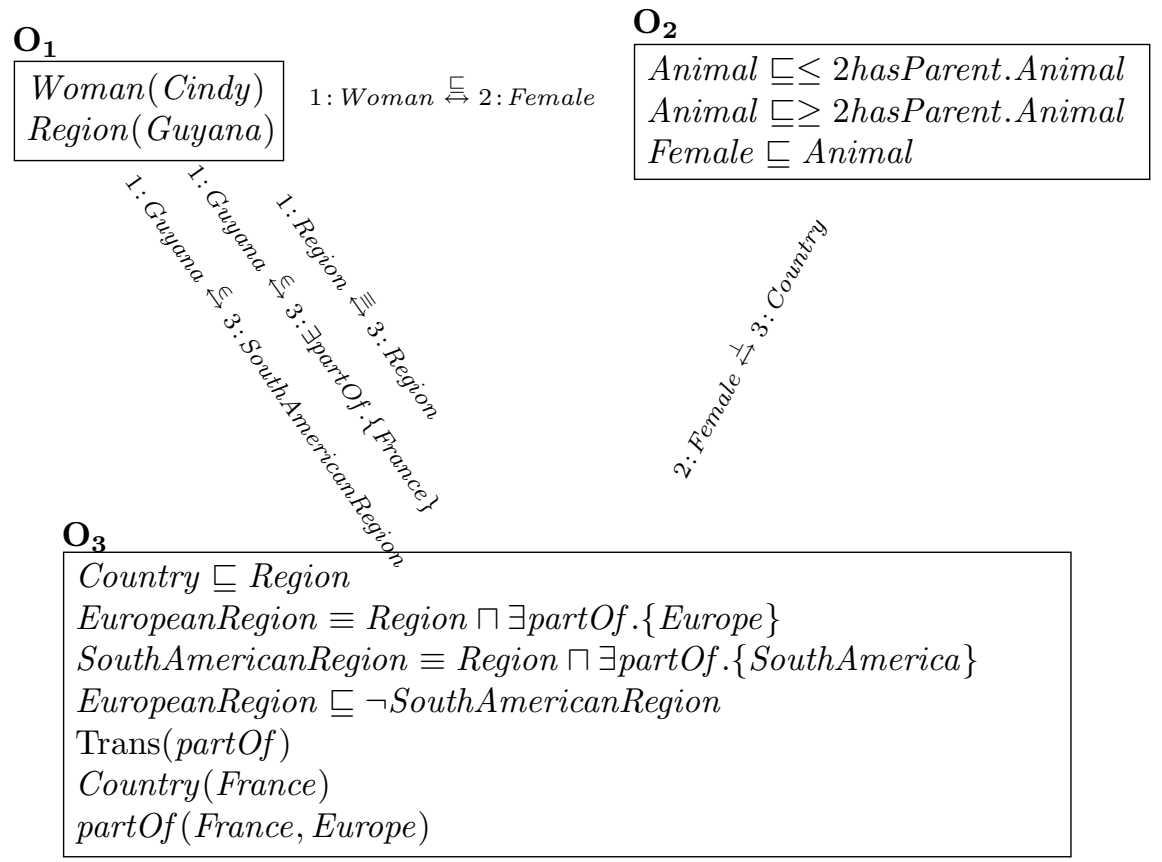

Figure 1: An example of distributed system. 
Formulas appearing between two ontologies denote correspondences between terms of different ontologies. Their formal meaning will be explained in details in the next section. Here, we just give an intuitive description of their sense. The correspondence between $O_{1}$ and $\mathrm{O}_{2}$ simply asserts that a Woman in $\mathrm{O}_{1}$ is a kind of Female in $\mathrm{O}_{2}$. The correspondence between $\mathrm{O}_{2}$ and $\mathrm{O}_{3}$ asserts the disjunctness of concepts Female and Country. Finally, the alignment between $O_{1}$ and $O_{3}$ denotes that a Region in $O_{1}$ defines the same thing as a Region in $O_{3}$; that Guyana is a part of France and Guyana is a region in South America.

A naive approach would simply consider correspondences as axioms adding supplementary information about the merged ontologies. While it is a very natural possibility, it leads to an unexpected inconsistency. Indeed, since Guyana is a part of France, and partOf is transitive, Guyana is also a part of Europe. Moreover, Guyana is a region, and therefore it ought to be European region. Besides, a correspondence tells that it is also a South American region. This is contradictory with the axiom of $O_{3}$ stating that European regions are disjoint with South American region.

Detecting inconsistencies is obviously not a bad thing. It serves to detect conceptual errors and revise the knowledge base accordingly. However, there are several notable facts that make it annoying in the context we are interested in. As pointed out in the introduction, each ontology is developed independently by very different parties. Consequently, semantic discrepancies are most likely to happen very often. Nonetheless, possible incompatibilities should not refrain one from using knowledge obtained through an aligned ontology. It is also important to consider that alignments can be provided by third party tools such as an alignment server that do not give greater importance to one or the other ontology. Finally, aligning ontology is a very difficult task and quality alignments should be used as much as possible. Therefore, we also believe that reusing alignments through operations like composition, union, intersection is crucial. For instance, according to the example, "Female subsumes Woman" and "Female is disjoint from Country" should entail "Woman is disjoint from Country".

\subsection{Requirements}

From this example we identify the following requirements:

- the overall consistency of the system should not be broken the slightest incompatibilities between local knowledge. This can be guaranteed by using a distributed semantics with several domains of interpretation, e.g., DDL, $\mathcal{E}$-connections, P-DL or IDDL;

- the semantics should comply with the fact that alignments are discovered from an outer point of view that encompasses both aligned ontologies. This eliminates DDL or $\mathcal{E}$-connections which express semantic relationships between ontologies according to a local ontology's point of view. P-DL is not relevant either, since its only way to relate ontologies is by importing terms. This is not enough for heterogeneous knowledge that we consider; 
- alignments should be shared across the system, so that they can be reused to build new correspondences out of existing ones, especially for transitive reusability of knowledge. Again, DDL or $\mathcal{E}$-connections do not offer this capability.

To better understand why other distributed formalisms do not comply with the identified requirements, we give a brief description of them in the next section.

\section{Related Work}

In this section we present several approaches to reasoning on a network of ontologies with alignments. These approaches result from different semantics admitted to alignments and from hypotheses about interpretation domains of the local ontologies.

\subsection{Merging-based approach}

This approach assumes that all modular ontologies and alignments are interpreted into the same domain $\Delta$. This means that local ontologies and alignments are considered as fragments of a global ontology simply obtained by merging them together. In this case, all reasoning on the local ontologies and alignments can be translated into those on the global ontology. This is exactly how OWL ontologies are treated [13. They are related thanks to import statement, and ontologies can relate local terms to imported ontology terms.

A straightforward merging gives this approach an advantage over the others: no new logic and algorithm for reasoning on modular ontologies and alignments is necessary. Moreover, efficient reasoners exist for such purpose [16, 1, 20, 19, 17. Using Description Logics as a formalism for modular reasoning is in particular advertised by [7, 6]. However, as pointed out in the example above, this approach would not be appropriate for reasoning on ontologies which are independently conceived and related by the means of alignments.

\section{$3.2 \mathcal{E}$-connections 8}

This approach relies on the assumption that all local ontologies are interpreted into disjoint domains. To express alignments, it introduces specific relations, namely links, between these domains. In order to express correspondences, new axioms have to be added to existing ontologies. For instance, if 1: FlatOwner, 2: Flat are concepts in ontology 1 and 2 respectively, owns is a link relation, then $1:$ FlatOwner $\sqsubseteq \exists$ owns.(2:Flat) is an axiom in ontology 1. [8] proposed an algorithm for reasoning on modular ontologies with alignments expressed via link relations. It enables a straightforward implementation based on an existing tableau reasoner.

There are two main disadvantages, w.r.t. our requirements. First, semantic relationship are asserted from one ontology point of view. So, an alignment server would not be able to properly construct links in $\mathcal{E}$-connections, except by being "tied" to a particular ontology. Second, the approach does not support transitive knowledge propagation among ontologies, which strongly restricts reusability of alignments accross the distributed system. 


\subsection{Distributed Description Logics (DDL) [4]}

DDL alignments are expressed via bridge rules. Each bridge rule simulates a cross-ontology concept subsumption or individual assertion which the logic used in ontologies allows for. For example, 1:Penguin $\sqsubseteq 2:$ Bird, 1:Penguin $\sqsubseteq 2: \neg F l y$ where 1 :Penguin is a concept in Ontology 1 and $2: \neg F l y, 2:$ Bird are concepts in Ontology 2. C-OWL [5] is an instantiation of the generic DDL semantics for distributed and contextualized OWL ontologies.

[18. proposed and implemented an algorithm for reasoning on ontologies with bridge rules of a very restricted form: no individual and constructed concept is allowed in bridge rules. The basic idea behind this algorithm is to translate the satisfiability problem with respect to a DDL ontology into several local satisfiability problems with respect to local ontologies. Despite the mentioned theoretical limitations, several works in [14] and [15] exploit successfully the reasoning algorithm implemented in DRAGO to improve or repair ontology mappings which are created by different matching systems without any human intervention.

\subsection{Package-based Description Logics (P-DL)}

P-DL [3] is useful for building modular ontologies in a homogeneous environment. Several ontologies (or "packages") are built separately, and connected to each others by explicitly stating that certain terms are imported from another package. There is no notion of alignments between packages, so that it would probably fail to represent the case study we are working on. However, different interpretations are assigned to different packages, and they only have to coincide on imported terms. Although authors have presented a tableaulike algorithm [2, it is restricted to the description logic $\mathcal{A L C}$ which has a relatively poor expressivity.

The limitations of each of these formalisms with respect to our requirements does not mean that the formalism we propose will replace or improve DDL, $\mathcal{E}$-connections or P-DL. Indeed, each one of these formalisms serves different purposes and we believe that IDDL is complementary to these. Moreover, it will be shown in Sect. 5 that several distributed formalisms can be used conjunctly.

\section{Integrated Distributed Description Logics (IDDL)}

Correlated Description Logics (IDDL) is in fact a new name for Integrated Distributed Description Logics (IDDL), first presented in [21. We decided to chose a new name to avoid confusion with Distributed Description Logics. IDDLis a formalism which inherits from both the field of Description Logics and from the analysis of distributed knowledge-based systems in 22 .

In a preliminary section, we define the notations we use to describe the syntax and semantics of classical description logics. Thereafter, we provide the definition of correspondence, alignment and distributed system, for which we define a semantics. 


\subsection{DL: syntax and semantics}

IDDL ontologies have the same syntax and semantics as in standard DL. More precisely, a DL ontology is composed of concepts, roles and individuals, as well as axioms built out of these elements. A concept is either a primitive concept $A$, or, given concepts $C$, $D$, role $R$, individuals $a_{1}, \ldots, a_{k}$, and natural number $n, \perp, \top, C \sqcup D, C \sqcap D, \exists R . C, \forall R . C, \leq n R . C$, $\geq n R . C, \neg C$ or $\left\{a_{1}, \ldots, a_{k}\right\}$. A role is either a primitive role $P$, or, given roles $R$ and $S$, $R \sqcup S, R \sqcap S, \neg R, R^{-}, R \circ S$ and $R^{+}$.

Interpretations are pairs $\left\langle\Delta^{I},{ }^{I}\right\rangle$, where $\Delta^{I}$ is a non-empty set (the domain of interpretation) and ${ }^{I}$ is the function of interpretation such that for all primitive concepts $A$, $A^{I} \subseteq \Delta^{I}$, for all primitive roles $P, P^{I} \subseteq \Delta^{I} \times \Delta^{I}$, and for all individuals $a, a^{I} \in \Delta^{I}$. Interpretations of complex concepts and roles is inductively defined by $\perp^{I}=\emptyset, \top^{I}=\Delta^{I}$, $(C \sqcup D)^{I}=C^{I} \cup D^{I},(C \sqcap D)^{I}=C^{I} \cap D^{I},(\exists R . C)^{I}=\left\{x \mid \exists y . y \in C^{I} \wedge\langle x, y\rangle \in R^{I}\right\}$, $(\forall R . C)^{I}=\left\{x \mid \forall y .\langle x, y\rangle \in R^{I} \Rightarrow y \in C^{I}\right\},(\leq n R . C)^{I}=\left\{x \mid \sharp\left\{y \in C^{I} \mid\langle x, y\rangle \in R^{I}\right\} \leq n\right\}$, $(\geq n R . C)^{I}=\left\{x \mid \sharp\left\{y \in C^{I} \mid\langle x, y\rangle \in R^{I}\right\} \geq n\right\},(\neg C)^{I}=\Delta^{I} \backslash C^{I},\left\{a_{1}, \ldots, a_{k}\right\}=\left\{a_{1}^{I}, \ldots, a_{k}^{I}\right\}$, $(R \sqcup S)^{I}=R^{I} \cup S^{I},(R \sqcap S)^{I}=R^{I} \cap S^{I},(\neg R)^{I}=\left(\Delta^{I} \times \Delta^{I}\right) \backslash R^{I},\left(R^{-}\right)^{I}=\left\{\langle x, y\rangle \mid\langle y, x\rangle \in R^{I}\right\}$, $(R \circ S)^{I}=\left\{\langle x, y\rangle \mid \exists z .\langle x, z\rangle \in R^{I} \wedge\langle z, y\rangle \in S^{I}\right\}$ and $\left(R^{+}\right)^{I}$ is the reflexive-transitive closure of $R^{I}$.

Axioms are either subsumption $C \sqsubseteq D$, sub-role axioms $R \sqsubseteq S$, instance assertions $C(a)$, role assertions $R(a, b)$ and individual identities $a=b$, where $C$ and $D$ are concepts, $R$ and $S$ are roles, and $a$ and $b$ are individuals. An interpretation $I$ satisfies axiom $C \sqsubseteq D$ iff $C^{I} \subseteq D^{I}$; it satisfies $R \sqsubseteq S$ iff $R^{I} \subseteq S^{I}$; it satisfies $C(a)$ iff $a^{I} \in C^{I}$; it satisfies $R(\bar{a}, b)$ iff $\left\langle a^{I}, b^{I}\right\rangle \in R^{I}$; and it satisfies $a=b$ iff $a^{I}=b^{I}$. When $I$ satisfies an axiom $\alpha$, it is denoted by $I \models \alpha$.

An ontology $O$ is composed of a set of terms (primitive concepts/roles and individuals) called the signature of $O$ and denoted by $\operatorname{Sig}(O)$, and a set of axioms denoted by $\operatorname{Ax}(O)$. An interpretation $I$ is a model of an ontology $O$ iff for all $\alpha \in \operatorname{Ax}(O), I \models \alpha$. In this case, we write $I \models O$. The set of all models of an ontology $O$ is denoted by $\operatorname{Mod}(O)$. A semantic consequence of an ontology $O$ is a formula $\alpha$ such that for all $I \in \operatorname{Mod}(O), I \models \alpha$.

\subsection{Distributed Systems}

A Distributed System (DS) is composed of a set of ontologies, interconnected by ontology alignments. An ontology alignment describes semantic relations between two ontologies.

\subsubsection{Syntax:}

An ontology alignment is composed of a set of correspondences. A correspondence can be seen as an axiom that asserts a relation between concepts, roles or individuals of two distinct ontologies. They are homologous to bridge rules in DDL. We use a notation similar to DDL in order to identify in which ontology a concept, role or individual is defined. If a concept/role/individual $E$ belongs to ontology $i$, then we write it $i: E$. The 6 possible types of correspondences between ontologies $i$ and $j$ are: 
Definition 1 (Correspondence) A correspondence between two ontologies $i$ and $j$ is one of the following formula:

- $i: C \stackrel{5}{\leftrightarrows} j: D$ is a cross-ontology concept subsumption;

- $i: R \stackrel{\sqsubseteq}{\longleftrightarrow} j: S$ is a cross-ontology role subsumption;

- $i: C \stackrel{\perp}{\longleftrightarrow} j: D$ is a cross-ontology concept disjunction;

- $i: R \stackrel{\perp}{\longleftrightarrow} j: S$ is a cross-ontology role disjunction;

- $i: a \stackrel{\in}{\longleftrightarrow} j: C$ is a cross-ontology membership;

- $i: a \stackrel{=}{\longleftrightarrow}: b$ is a cross-ontology identity.

An ontology alignment is simply a set of correspondences. Together with DL ontologies, they form the components of a Distributed System in IDDL.

Definition 2 (Distributed System) A Distributed System (DS for short), is a pair $\langle\mathbf{O}, \mathbf{A}\rangle$ such that $\mathbf{O}$ is a set of ontologies, and $\mathbf{A}=\left(A_{i j}\right)_{i, j \in \mathbf{O}}$ is a family of alignments relating ontologies of $\mathbf{O}^{1}$

\subsubsection{Semantics}

Distributed systems semantics depends on local semantics, but does not interfere with it. A standard DL ontology can be straightforwardly used in IDDL system. Informally, interpreting a IDDL system consists in assigning a standard DL interpretation to each local ontology, then correlating the domains of interpretation thanks to what we call an equalizing function.

Definition 3 (equalizing function) Given a family of local interpretations $\mathbf{I}$, an equalizing function $\epsilon$ is a family of functions indexed by $\mathbf{I}$ such that for all $I_{i} \in \mathbf{I}, \epsilon_{i}: \Delta^{I_{i}} \rightarrow \Delta_{\epsilon}$ where $\Delta_{\epsilon}$ is called the global domain of interpretation of $\epsilon$.

A distributed interpretation assigns a standard DL interpretation to each ontology in the system, as well as an equalizing function that correlate local knowledge into a global domain of interpretation.

Definition 4 (Distributed interpretation) Let $S=\langle\mathbf{O}, \mathbf{A}\rangle$ be a $D S . A$ distributed interpretation of $S$ is a pair $\langle\mathbf{I}, \epsilon\rangle$ where $\mathbf{I}$ is a family of interpretations indexed by $\mathbf{O}, \epsilon$ is an equalizing function for $\mathbf{I}$, such that for all $i \in \mathbf{O}, I_{i}$ interprets $i$ and $\epsilon_{i}: \Delta^{I_{i}} \rightarrow \Delta_{\epsilon}$.

While local satisfiability is the same as standard DL, correspondence satisfaction involves the equalizing function.

\footnotetext{
${ }^{1}$ We consistently use bold face to denote a mathematical family of elements. So, $\mathbf{O}$ denotes $\left(O_{i}\right)_{i \in I}$ where $I$ is a set of indices.
} 
Definition 5 (Satisfaction of a correspondence) Let $S$ be a DS, and $i, j$ two ontologies of $S$. Let $\mathcal{I}=\langle\mathbf{I}, \epsilon\rangle$ be a distributed interpretation. We define satisfaction of a correspondence $c$ (denoted by $\left.\left.\mathcal{I}\right|_{d} c\right)$ as follows:

$$
\begin{aligned}
& \mathcal{I} \models_{d} i: C \stackrel{\sqsubseteq}{\longleftrightarrow} j: D \quad \text { iff } \quad \epsilon_{i}\left(C^{I_{i}}\right) \subseteq \epsilon_{j}\left(D^{I_{j}}\right) \\
& \mathcal{I} \models_{d} i: R \stackrel{\sqsubseteq}{\leftrightarrows} j: S \quad \text { iff } \quad \epsilon_{i}\left(R^{I_{i}}\right) \subseteq \epsilon_{j}\left(S^{I_{j}}\right) \\
& \mathcal{I} \models{ }_{d} i: C \stackrel{\perp}{\longleftrightarrow} j: D \quad \text { iff } \quad \epsilon_{i}\left(C^{I_{i}}\right) \cap \epsilon_{j}\left(D^{I_{j}}\right)=\emptyset \\
& \mathcal{I} \models_{d} i: R \stackrel{\perp}{\longleftrightarrow} j: S \quad \text { iff } \quad \epsilon_{i}\left(R^{I_{i}}\right) \cap \epsilon_{j}\left(S^{I_{j}}\right)=\emptyset \\
& \mathcal{I} \models_{d} i: a \stackrel{\epsilon}{\longleftrightarrow} j: C \quad \text { iff } \quad \epsilon_{i}\left(a^{I_{i}}\right) \in \epsilon_{j}\left(C^{I_{j}}\right) \\
& \mathcal{I} \models_{d} i: a \stackrel{=}{\longleftrightarrow} j: b \quad \text { iff } \quad \epsilon_{i}\left(a^{I_{i}}\right)=\epsilon_{j}\left(b^{I_{j}}\right)
\end{aligned}
$$

Additionally, for all local formula $i: \phi, \mathcal{I} \models{ }_{d} i: \phi$ iff $I_{i} \models \phi$ (i.e., local satisfaction implies global satisfaction). A distributed interpretation $\mathcal{I}$ satisfies an alignment $A$ iff it satisfies all correspondences of $A$ (denoted by $\mathcal{I} \models_{d} A$ ) and it satisfies an ontology $O_{i}$ iff it satisfies all axioms of $O_{i}$ (denoted by $\mathcal{I} \models{ }_{d} O_{i}$ ). When all ontologies and all alignments are satisfied, the DS is satisfied by the distributed interpretation. In which case we call this interpretation a model of the system.

Definition 6 (Model of a DS) Let $S=\langle\mathbf{O}, \mathbf{A}\rangle$ be a DS. A distributed interpretation $\mathcal{I}$ is a model of $S$ (denoted by $\mathcal{I} \models{ }_{d} S$ ), iff:

- for all $O_{i} \in \mathbf{O}, \mathcal{I} \models{ }_{d} O_{i}$;

- for all $A_{i j} \in \mathbf{A}, \mathcal{I} \models{ }_{d} A_{i j}$.

The set of all models of a DS is denoted by $\operatorname{Mod}(S)$. A formula $\alpha$ is a consequence of a DS $\left(S \models_{d} \alpha\right)$ iff $\forall \mathcal{M} \in \operatorname{Mod}(S), \mathcal{M} \models_{d} \alpha$. This model-theoretic semantics offers special challenge to the reasoning infrastructure, that we discuss in next section.

\section{Reasoning in IDDL with concept correspondences}

In this section, we investigate a reasoning procedure for checking whether or not $S=\langle\mathbf{O}, \mathbf{A}\rangle$ is consistent, in the case when only concepts are put in correspondences. Role correspondences are considered in Sect. 8 ,

We can reduce the problem of entailment $S \models_{d} \alpha$ to deciding (in)consistency of a DS

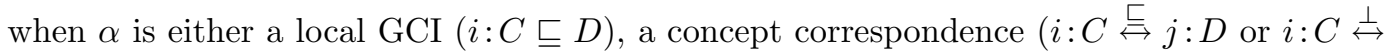
$j: D$ ) or a local ABox assertion $(i: C(a))$. Local entailment reduction is straightforward. However, correspondence entailment like $S \models_{d} i: C \stackrel{\sqsubseteq}{\leftrightarrows} j: D$ is equivalent to the inconsistency of $S \cup\{i:\{a\} \stackrel{\sqsubseteq}{\Leftrightarrow} i: C\} \cup\{i:\{a\} \stackrel{\perp}{\Leftrightarrow} j: D\}$, where $a$ is a new individual name added to ontology $O_{i}$. 
When ontologies are correlated with alignments, new deductions may occur. Indeed, cross-ontology knowledge interacts with local knowledge. Moreover, knowledge from one ontology may influence knowledge from another ontology. Besides, local knowledge would also induce cross-ontology knowledge (i.e., alignments). And finally, deductions can be made with and about the alignments alone.

In fact, the difficulty of reasoning in IDDL resides in determining what knowledge propagates from local domains to global domain, or from global to local domains. In fact, we will show that, in the restricted case when only concept correspondences are allowed, only unsatisfiability and non-unsatiafiability of concepts are propagated.

Example 1 Let $O_{1}=\left\{C_{1} \equiv A_{1} \sqcap B_{1}\right\}, O_{2}=\left\{A_{2} \sqsubseteq \top, B_{2} \sqsubseteq \top\right\}$ and alignment $A_{12}=$ $\left\{A_{1} \sqsubseteq A_{2}, B_{1} \perp A_{2}, C_{1} \sqsupseteq B_{2}\right\}$.

We have $A_{12} \models A_{1} \perp B_{1}$. If $A_{1} \perp B_{1}$ is added to $O_{1}$ (by the intuition ??) then $C_{1}$ becomes unsatisfiable in $O_{1}$. From the correspondence $C_{1} \sqsupseteq B_{2}$, it follows that $B_{2}$ is unsatisfiable in $\mathrm{O}_{2}$ as well.

The example above shows that reasoning on IDDL systems is not trivial and the existing algorithms for reasoning on DL-based ontologies (e.g., tableau algorithms) can not be directly used.

The principle behind the algorithm is based on the fact that correspondences are similar to axioms, and alignments resembles ontologies. In fact, an alignment represents an ontology which would be interpreted in the global domain of interpretation (see Def. 4 ). In this algorithm, the alignments will be translated into an ontology (the global ontology). However, this is not enough to check global consistency because local knowledge influences global reasoning. So, the idea consists in extending the global ontology together with the local ontologies by adding specific axioms wich represent knwoledge propagated through the distributed system.

As a matter of fact, if correspondences are restricted to cross-ontology concept subsumption or disjointness, only concept unsatisfiability and concept non-emptiness can propogate. Indeed, if a concept is locally interpreted as empty, then its image via the equalizing function is empty too. Conversely, a non-empty set has a non-empty image through $\epsilon$.

Unfortunately, it is not possible to propagate knowledge by analysing ontologies one by one. A subtle combination of several ontologies and alignments can impose unsatisfiability of a locally satisfiable concept (see Ex. 1).

In order to be certain that all concept unsatisfiability and non-emptiness are propagated, our algorithm exhaustively tests each combination of concept unsatisfiability and non-emptiness by explicitely adding these facts, and propagating them accordingly.

In the sequel, we introduce the construction of extended ontologies which are built from $\mathbf{A}$ and $\mathbf{O}$ and we show that the consistency of a IDDL system $S$ is equivalent to the existence of such extended ontologies such that they are consistent. 


\subsection{Configurations and extended ontologies}

This section provides the formal definitions which will finally lead to the construction of the extended ontologies which have been mentionned above. A configuration determines whether certain well chosen concepts in a vocabulary are unsatisfiable or non-empty. In our specific case, the vocabulary in question is defined by the correspondences. It will be proven that it is sufficient to consider concepts appearing in correspondences when dealing with knowledge propagation in IDDL.

Concepts occurring as the left or right side of correspondences in alignments constitute the vocabulary of an alignment ontology, namely global vocabulary. This vocabulary consists of local vocabularies which are originated from local ontologies. The following definitions introduce formally the construction of these elements which will be used for formulating axioms or assertions in alignment and extended ontologies.

Definition 7 (Local vocabulary) Let $S=\langle\mathbf{O}, \mathbf{A}\rangle$ be a $D S$. We denote by $\mathscr{C}_{i}$ the set that includes the top concept $T$ and all (primitive or complex) concepts that appear in the left side of correspondences in $A_{i j}$ or in the right side of correspondences in $A_{j i}$.

Definition 8 (Global vocabulary) Let $S=\langle\mathbf{O}, \mathbf{A}\rangle$ be a DS. The set of global concept names of $S$ is $\mathscr{C}=\bigcup_{i \in \mathbf{O}}\left\{i: C \mid C \in \mathscr{C}_{i}\right\}$. When $w \subseteq \mathscr{C}_{i}$, we denote by $\widehat{w}$ the set $\{i: C \mid C \in w\}$ of (global) concept names. When $W \subseteq \mathscr{C}$, we denote by $\left.W\right|_{i}$ the set $\left\{C \in \mathscr{C}_{i} \mid i: C \in W\right\}$.

Considering Example1, we have local vocabularies $\mathscr{C}_{1}=\left\{A_{1}, B_{1}, C_{1}, \top_{1}\right\}, \mathscr{C}_{2}=\left\{A_{2}, B_{2}, \top_{2}\right\}$, $\mathscr{R}_{1}=\mathscr{R}_{2}=\emptyset$, and global vocabulary $\mathscr{C}=\left\{A_{1}, B_{1}, C_{1}, \top_{1}, A_{2}, B_{2}, \top_{2}\right\}, \mathscr{R}=\emptyset$.

In the algorithm, we do not provide a configuration for the set of global or local concepts directly. In fact, a configuration defines the non-emptiness of some particular concepts defined as follows.

Definition 9 Let $T$ be a set of concepts (primitive or complex). For each non empty subset $W \subseteq T$, we define the concept $C_{W}^{T}:=\left(\prod_{X \in W} X \sqcap \prod_{X^{\prime} \in T \backslash W} \neg X^{\prime}\right)$.

When there is no ambiguity, we denote by $C_{W}$ the concept $C_{W}^{\mathscr{C}}$ defined above when $\mathscr{C}$ is the global vocabulary of a distributed system $S$.

Roughly speaking, a configuration of models for an ontology over a set $\mathscr{C}$ of concepts can be considered as an equivalence class of models which determines a partition of an interpretation domain by interpretations of the concepts in $\mathscr{C}$. Two models belong to a configuration (equivalence class) if for each subset $W \subseteq \mathscr{C}$ the two interpretations of the concept $C_{W}^{\mathscr{C}}$ (introduced by Def. 9 under these models are empty or not, simultaneously. If $\mathscr{C}$ is global vocabulary then the knowledge about the unsatisfiability or non-unsatisfiability (non-emptiness) of $C_{W}^{\mathscr{C}}$ must be propagated from alignment $\mathbf{A}$ to local ontologies $\left\{\mathbf{O}_{i}\right\}$. This knowledge can be directly expressed as in Def. 10 . 
Definition 10 (Global configuration) Let $S$ be a DS with a set of global concept names $\mathscr{C}$. A global configuration of $S$ is a subset $\Omega$ of $2^{\mathscr{C}}$.

We have prepared necessary elements for constructing the so-called alignment ontology. This ontology "axiomatize" the alignments, which represent inter-ontology knowledge. Apart from axioms expressing correspondences in alignments, an alignment ontology includes additional axioms or assertions representing the global configuration.

Definition 11 (Alignment ontology) Let $S=\langle\mathbf{O}, \mathbf{A}\rangle$ be a DS. Let $\Omega$ be a global configuration of $S$. The alignment ontology with respect to $\Omega$ is an ontology $\widehat{\mathbf{A}}_{\Omega}$ defined as follows:

1. for each $i, j \in \mathbf{O}$, if $i: C \stackrel{\sqsubseteq}{\leftrightarrows} j: D$ (resp. $i: C \stackrel{\perp}{\leftrightarrows} j: D)$ is a concept correspondence in $\mathbf{A}$ then $i: C \sqsubseteq j: D$ (resp. $i: C \sqsubseteq \neg j: D)$ is an axiom of $\widehat{\mathbf{A}}_{\Omega}$;

2. for each $W \in \Omega, C_{W} \equiv\left\{a_{W}\right\}$ is an axiom of $\widehat{\mathbf{A}}_{\Omega}$ where $a_{W}$ is a new individual name;

3. for each $W \notin \Omega, C_{W} \sqsubseteq \perp$ is an axiom of $\widehat{\mathbf{A}}_{\Omega}$.

Reconsidering Example 1, if we pick, for instance, the configuration $\Omega=2^{\mathscr{C}}$ to build an alignment ontology $\widehat{\mathbf{A}}$ according to Def. 11 , then $\widehat{\mathbf{A}}$ is inconsistent.

The construction of local configurations is very similar to that of global configuration except that compatibility of local configurations with a given global configuration must be taken into account. This compatibility results from the semantics of IDDL system relying on equalizing functions, which imposes that if the image of a set under an equalizing function is not empty then that set must be not empty.

Definition 12 (Local configuration) Let $S=\langle\mathbf{O}, \mathbf{A}\rangle$ be a DS. Let $\Omega$ be a global configuration of $S$. For each $O_{i} \in \mathbf{O}$, we define a local configuration of $O_{i}$ with respect to $\Omega$ as a subset $\Omega^{i}$ of $\mathscr{Q}_{i}^{\mathscr{C}}$. Moreover, if $w \in \Omega^{i}$ then there must exist $W \in \Omega$ such that $\widehat{w} \subseteq W$.

As discussed at the beginning of this section, knowledge propagation from alignments to local ontologies is crucial to the construction of extended ontologies which preserve the consistency of a IDDL system. A global configuration $\Omega$ and a local configuration $\Omega^{i}$ which is compatible with $\Omega$ provide necessary elements to define such extended ontologies. The following definition describes how to propagate knowledge from alignments to local ontologies through the determined configurations.

Definition 13 (Extended ontologies) Let $S=\langle\mathbf{O}, \mathbf{A}\rangle$ be a DS. Let $\Omega$ be a global configuration of $S$. For each $O_{i} \in \mathbf{O}$, let $\Omega^{i}$ be a local configuration with respect to $\Omega$. The extended ontology $\widehat{O}_{\Omega^{i}}$ with respect to $\Omega^{i}$ and $\Omega$ is defined as follows:

1. $O_{i} \subseteq \widehat{O}_{\Omega^{i}}$;

2. for each $w \in \Omega^{i}, C_{w}^{\mathcal{C}_{i}}\left(b_{w}\right)$ is an axiom of $\widehat{O}_{\Omega^{i}}$, where $b_{w}$ is a new individual name; 
3. for each $w \notin \Omega^{i}, C_{w}^{\mathcal{C}_{i}} \sqsubseteq \perp$ is an axiom of $\widehat{O}_{\Omega^{i}}$;

4. for each $W \in \Omega$ and for each $\left.X \in W\right|_{i}$, we define a new concept $C_{W}^{X}$ for ontology $\widehat{O}_{\Omega^{i}}$ such that:

(a) $C_{W}^{X} \sqsubseteq X \sqcap \prod_{\left.X^{\prime} \in \mathscr{C}_{i} \backslash W\right|_{i}} \neg X^{\prime}$ is an axiom of $\widehat{O}_{\Omega^{i}}$;

(b) $C_{W}^{X}\left(b_{W}^{X}\right)$ is an axiom of $\widehat{O}_{\Omega^{i}}$ with $b_{W}^{X}$ a new individual name in $\widehat{O}_{\Omega^{i}}$;

(c) $C_{W}^{X} \sqsubseteq C_{W}^{\top}$ is an axiom of $\widehat{O}_{\Omega^{i}}$;

5. for each $W, W^{\prime} \subseteq \mathscr{C}$ such that $W \neq W^{\prime}, C_{W}^{\top} \sqsubseteq \neg C_{W^{\prime}}^{\top}$ is an axiom of $\widehat{O}_{\Omega^{i}}$.

The concept $C_{W}^{X}$ represents the set of elements in local concept $X$ which have their global counterparts in the global concept $C_{W}$. Notice that the propagation of the knowledge through a global configuration is not straightforward. The non-emptiness expressed by the assertion $C_{W}\left(a_{W}\right)$ must not be directly propagated. The reason for this is that the nonemptiness of $\epsilon_{i}\left(\mathcal{I}_{i}\left(C_{W}\right)\right)$ with a local interpretation $\mathcal{I}_{i}$ and an equalizing function $\epsilon_{i}$, does not imply the non-emptiness of $\mathcal{I}_{i}\left(C_{\left.W\right|_{i}}^{\mathscr{C}_{i}}\right)$. Consequently, the decomposition of the concept $C_{W}$ for the propagation as described in the item 4 in Def. 13 is necessary.

The following theorem establishes the most important result in the present section. It asserts that a IDDL system can be translated into an alignment ontology and extended ontologies that preserve the semantics of the IDDL system.

\section{Theorem and proof}

Theorem 1 (DS Consistency) Let $S=\langle\mathbf{O}, \mathbf{A}\rangle$ be a DS. $S$ is consistent if and only if there exist a global configuration $\Omega$ of $S$ and a local configuration $\Omega^{i}$ for each $O_{i} \in \mathbf{O}$ with respect to $\Omega$ such that the alignment ontology $\widehat{\mathbf{A}}_{\Omega}$ and the extended local ontologies $\left\{\widehat{O}_{\Omega^{i}}\right\}$ as defined in Def. 11 and Def. 13 are consistent.

Proof: The proof of this theorem is quite complex, so we will separate into sections and subsections, with intermediary lemmas. Since the theorem asserts an equivalence (if and only if) we will separate the proof in two big steps, one for the "if-direction" (i.e., $\Leftarrow$ ) in Sect. 6.1, and one for the "only-if-direction" (i.e., $\Rightarrow$ ) in Sect. 6.2.

\subsection{If-direction $\Leftarrow$}

In this part, we assume the existence of a global configuration $\Omega$ and local configurations $\Omega^{i}$ such that $\widehat{\mathbf{A}}_{\Omega}$ and $\widehat{O}_{\Omega^{i}}$ are consistent. We prove that $S$ is consistent in this case by asserting the existence of a model $\mathcal{I}_{A}$ of $\widehat{\mathbf{A}}_{\Omega}$ and models $\mathcal{I}_{i}$ of $\widehat{O}_{\Omega^{i}}$ from which we build a distributed model of $S$. More precisely, the models of $\widehat{O}_{\Omega^{i}}$ are also models of $O_{i}$. So, building a model of $S$ amounts to building an equalizing function $\epsilon$ for $\mathbf{I}=\left(\mathcal{I}_{i}\right)_{i \in \mathbf{O}}$ such that $\langle\mathbf{I}, \epsilon\rangle \models S$. We 


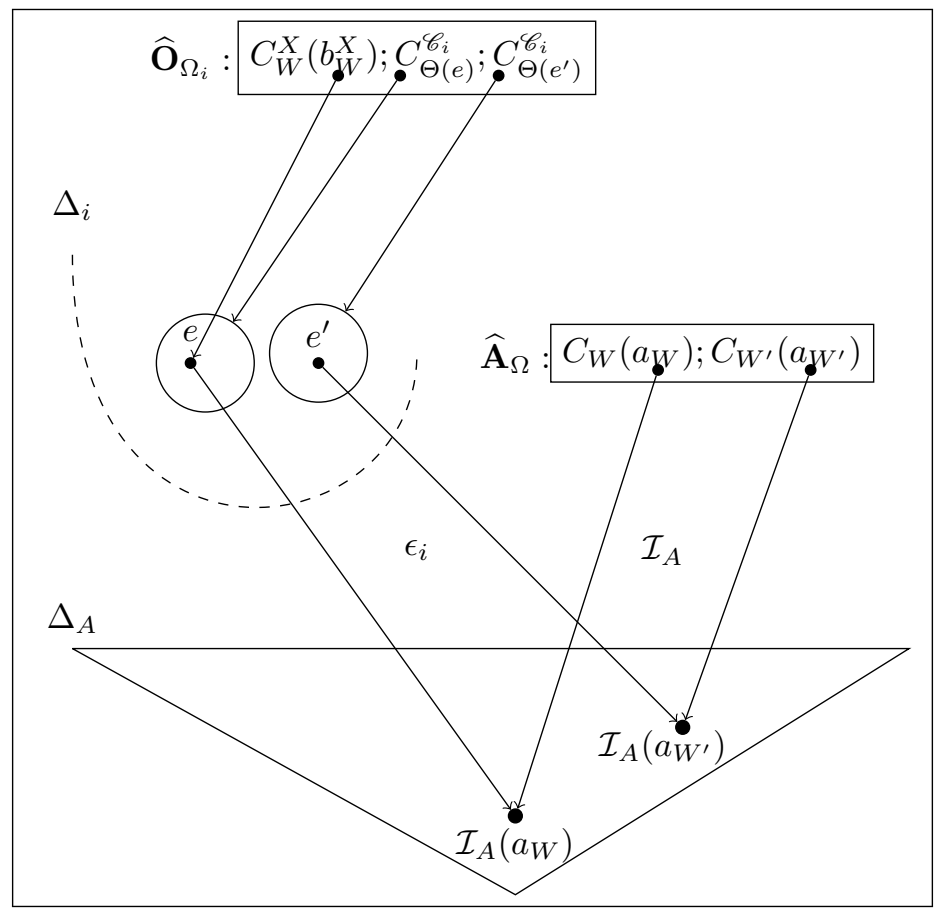

Figure 2: Construction of $\epsilon_{i}$ from $\mathcal{I}_{i}$ and $\mathcal{I}_{A}$. For each $e=\mathcal{I}_{i}\left(b_{W}^{X}\right)$ or $e \in \mathcal{I}_{i}\left(C_{\Theta(e)}^{\mathscr{C}_{i}}\right)$ we can find an individual $a_{W}$ in $\widehat{\mathbf{A}}_{\Omega}$ such that $\epsilon_{i}(e)$ is assigned to $\mathcal{I}_{A}\left(a_{W}\right)$.

separate this process in the following steps: first, we explain the construction of $\epsilon_{i}$ according to $\mathcal{I}_{A}$ and $\mathcal{I}_{i}$; second, we prove an important lemma which helps proving that we have built a model of $S$; third, the actual proof of the satisfaction of $S$ is decomposed in proofs of the satisfaction of the various kinds of correspondences.

Before continuing the proof, we define the function $\Theta: \Delta_{i} \rightarrow \mathscr{2}_{i}^{\mathscr{C}}$ which maps each element $e \in \Delta_{i}$ to the set of concepts in $\mathscr{C}_{i}$ which have an interpretation including $e$ (i.e., $\left.\Theta(e)=\left\{X \in \mathscr{C}_{i} \mid e \in \mathcal{I}_{i}(X)\right\}\right)$. Since $\top \in \mathscr{C}_{i}$ hence $\Theta(e) \neq \emptyset$. This implies that $C_{\Theta(e)}^{\mathscr{C}_{i}} \sqsubseteq$ $\perp \notin \widehat{O}_{\Omega^{i}}$, i.e., $\Theta(e) \in \Omega^{i}$.

\subsubsection{Building the equalizing function.}

According to the hypothesis in this section, there exists a model $\left\langle\Delta_{A},{ }^{\mathcal{I}_{A}}\right\rangle$ of $\widehat{\mathbf{A}}_{\Omega}$ and a model $\left\langle\Delta_{i},{ }^{\mathcal{I}_{i}}\right\rangle$ of $\widehat{O}_{\Omega^{i}}$ for each $i \in \mathbf{O}$.

We now define for all $i$ a function $\epsilon_{i}$ from $\Delta_{i}$ into $\Delta_{A}$ (see Fig. 2). Let $e \in \Delta_{i}$.

According to Def. 12 and the definition of $\Theta(e)$, there exists $W \in \Omega$ such that $\widehat{\Theta(e)} \subseteq W$, i.e., $\left.\Theta(e) \subseteq W\right|_{i} .(*)$ 
Then, we distinguish the two following cases.

1. If there exists $W^{\prime} \in \Omega$ and $e \in \mathcal{I}_{i}\left(C_{W^{\prime}}^{\top}\right)$ then we define $\epsilon_{i}(e)=\mathcal{I}_{A}\left(a_{W^{\prime}}\right)$ with $C_{W^{\prime}}^{\top}$ defined according to item 4 in Def. 13

2. In any other situation, we define $\epsilon_{i}(e)=\mathcal{I}_{A}\left(a_{W}\right)$ (with $W$ introduced above at $(*)$ ).

This is a valid definition of function $\epsilon_{i}$, because if $e \in \mathcal{I}_{i}\left(C_{W^{\prime}}^{\top}\right)$ and $e \in \mathcal{I}_{i}\left(C_{W^{\prime \prime}}^{\top}\right)$ then $W^{\prime}=W^{\prime \prime}$ thanks to item 5 of Def. 13 .

\subsubsection{Auxiliary lemma.}

The following lemma asserts that each element $e$ of a local domain of interpretation $\Delta_{i}$ can be associated to a set of global concepts $W \in \Omega$ such that $\left.\Theta(e) \subseteq W\right|_{i}$ and for which $\epsilon_{i}(e)=\mathcal{I}_{A}\left(a_{W}\right)$. More informally, this lemma serves to show that the local structures of class containment and membership are preserved in the alignment ontology.

Lemma 1 For each $e \in \Delta_{i}$ there exists $W \in \Omega$ such that $\left.\Theta(e) \subseteq W\right|_{i}$ and $\epsilon_{i}(e)=\mathcal{I}_{A}\left(a_{W}\right)$.

Proof: To prove Lem. 1, we consider the following two cases according to the definition of $\epsilon_{i}$ :

1. If there exists $W^{\prime} \in \Omega$ and $e \in \mathcal{I}_{i}\left(C_{W^{\prime}}^{\top}\right)$ then we have $\epsilon_{i}(e)=\mathcal{I}_{A}\left(a_{W^{\prime}}\right)$. Therefore, $e \in \bigcap_{\left.X \in \mathscr{C}_{i} \backslash W^{\prime}\right|_{i}} \Delta_{i} \backslash \mathcal{I}_{i}(X)$. This implies that $\left.\Theta(e) \subseteq W^{\prime}\right|_{i}$ (otherwise, $e$ would be in $\mathcal{I}_{i}(\neg X)$ for some $\left.X \in \Theta(e)\right)$.

2. In any other cases, the property is trivially satisfied.

\subsubsection{Satisfaction of the system.}

Since $O_{i}$ is included in $\widehat{O}_{\Omega^{i}}$ for all $i$, we already have models for all $O_{i}$. We now show that the conditions in Def. 5 are satisfied with functions $\left\{\mathcal{I}_{i}\right\}$ and $\left\{\epsilon_{i}\right\}$ (see Fig. 3). Since concepts can be nominal, cross-ontology membership and cross-ontology identity can be translated into cross-ontology concept subsumption and cross-ontology concept equivalence. These proofs are a bit technical, but quite straightforward. The only trick resides in the appropriate use of the above lemma.

Cross-ontology concept subsumption $\left(i: C \stackrel{\sqsubseteq}{\leftrightarrows}: D \in A_{i j} \Longrightarrow \epsilon_{i}\left(\mathcal{I}_{i}(C)\right) \subseteq \epsilon_{j}\left(\mathcal{I}_{j}(D)\right)\right)$. Let us assume that $i: C \stackrel{\sqsubseteq}{\leftrightarrows} j: D \in A_{i j}$. Let $x \in \epsilon_{i}\left(\mathcal{I}_{i}(C)\right)$. There exists $e \in \mathcal{I}_{i}(C)$ such that $\epsilon_{i}(e)=x$. From Lem. 1, it follows that there exists $W \in \Omega$ such that $\left.\Theta(e) \subseteq W\right|_{i}$ and $\epsilon_{i}(e)=\mathcal{I}_{A}\left(a_{W}\right)$. Moreover, since $\mathcal{I}_{A}$ is a model for $\widehat{\mathbf{A}}_{\Omega}$ and $i: C \sqsubseteq j: D \in \widehat{\mathbf{A}}_{\Omega}$ and $C_{W} \equiv\left\{a_{W}\right\} \in \widehat{\mathbf{A}}_{\Omega}$ (according to Def. 11 hence $\mathcal{I}_{A}\left(a_{W}\right) \in \mathcal{I}_{A}(j: D)$. By the construction 


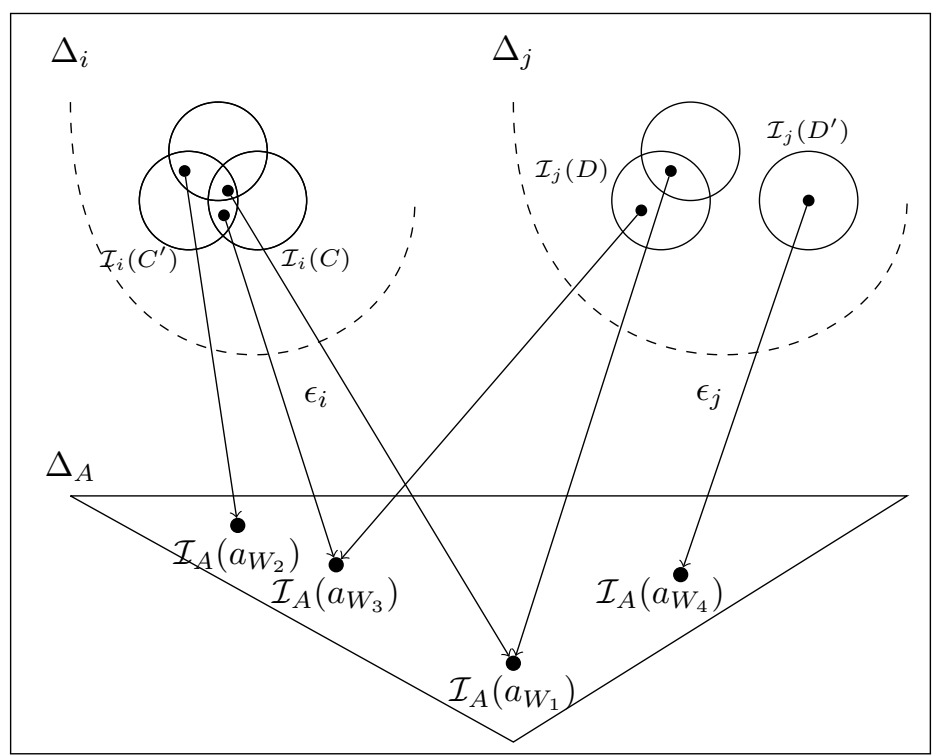

Figure 3: The figure illustrates $\left(i: C \stackrel{\sqsubseteq}{\Leftrightarrow} j: D \in A_{i j} \Longrightarrow \epsilon_{i}\left(\mathcal{I}_{i}(C)\right) \subseteq \epsilon_{j}\left(\mathcal{I}_{j}(D)\right)\right)$ and $\left(i: C^{\prime} \stackrel{\perp}{\leftrightarrow} j: D^{\prime} \in A_{i j} \Longrightarrow \epsilon_{i}\left(\mathcal{I}_{i}\left(C^{\prime}\right)\right) \cap \epsilon_{j}\left(\mathcal{I}_{j}\left(D^{\prime}\right)\right)=\emptyset\right)$

of $C_{W}$ and since $C_{W} \equiv\left\{a_{W}\right\}$ is in $\widehat{\mathbf{A}}_{\Omega}$, we have $j: D \in W$ (otherwise, $\neg j: D$ must be included in $C_{W}$, which contradicts $\left.\mathcal{I}_{A}\left(a_{W}\right) \in \mathcal{I}_{A}(j: D)\right)$. By Def. 13 we have $\widehat{O}_{\Omega^{j}} \models$ $\left(D \sqcap \prod_{\left.X^{\prime} \in \mathscr{C}_{j} \backslash W\right|_{j}} \neg X^{\prime}\right)\left(b_{W}^{D}\right)$. This implies that $\mathcal{I}_{j}\left(b_{W}^{D}\right) \in \mathcal{I}_{j}(D)$. Moreover, $\widehat{O}_{\Omega^{j}} \models C_{W}^{\top}\left(b_{W}^{D}\right)$.

From the definition of $\epsilon_{j}$ we obtain $\epsilon_{j}\left(\mathcal{I}_{j}\left(b_{W}^{D}\right)\right)=\mathcal{I}_{A}\left(a_{W}\right)$. This implies that $x=\epsilon_{j}\left(\mathcal{I}_{j}\left(b_{W}^{D}\right)\right)$, and since $\mathcal{I}_{j}\left(b_{W}^{D}\right) \in \mathcal{I}_{j}(D), x \in \epsilon_{j}\left(\mathcal{I}_{j}(D)\right)$. As a result, $\epsilon_{i}\left(\mathcal{I}_{i}(C)\right) \subseteq \epsilon_{j}\left(\mathcal{I}_{j}(D)\right)$.

Cross-ontology concept disjointness $\left(i: C \stackrel{\doteq}{\Leftrightarrow} j: D \in A_{i j} \Longrightarrow \epsilon_{i}\left(\mathcal{I}_{i}(C)\right) \cap \epsilon_{j}\left(\mathcal{I}_{j}(D)\right)=\emptyset\right)$. Let us assume that $i: C \stackrel{\perp}{\Leftrightarrow} j: D \in A_{i j}$. Let $x \in \epsilon_{i}\left(\mathcal{I}_{i}(C)\right)$. There exists $e \in \mathcal{I}_{i}(C)$ such that $\epsilon_{i}(e)=x$. From Lem. 1, it follows that there exists $W \in \Omega$ such that $\left.\Theta(e) \subseteq W\right|_{i}$ and $\epsilon_{i}(e)=\mathcal{I}_{A}\left(a_{W}\right)$. Moreover, since $\mathcal{I}_{A}$ is a model for $\widehat{\mathbf{A}}_{\Omega}$ and $i: C \sqsubseteq \neg j: D \in \widehat{\mathbf{A}}_{\Omega}$ and $C_{W} \equiv\left\{a_{W}\right\} \in \widehat{\mathbf{A}}_{\Omega}$ we have $\mathcal{I}_{A}\left(a_{W}\right) \notin \mathcal{I}_{A}(j: D)$. $\left({ }^{* *}\right)$

Furthermore, for all $f \in \mathcal{I}_{j}(D)$, by Lem. 1 1 there exists $W^{\prime} \in \Omega$ such that $\epsilon_{j}(f)=$ $\mathcal{I}_{A}\left(a_{W^{\prime}}\right)$ and $\left.\Theta(f) \subseteq W^{\prime}\right|_{j}$, so $\mathcal{I}_{A}\left(a_{W^{\prime}}\right) \in \mathcal{I}_{A}(j: D)$. From this and $\left({ }^{* *}\right)$, we obtain $\mathcal{I}_{A}\left(a_{W}\right) \neq \mathcal{I}_{A}\left(a_{W^{\prime}}\right)$. Thus, $\epsilon_{i}(e) \neq \epsilon_{j}(f)$ for all $f \in \mathcal{I}_{j}(D)$. As a result $\epsilon_{i}\left(\mathcal{I}_{i}(C)\right) \cap$ $\epsilon_{j}\left(\mathcal{I}_{j}(D)\right)=\emptyset$.

Thus, the distributed interpretation $\left\langle\left(\mathcal{I}_{i}\right),\left(\epsilon_{i}\right)\right\rangle$ satisfies the system $S$. Therefore $S$ is consistent. 


\subsection{Only-if-direction}

In this section, we assume that $S$ is consistent. Therefore, there exists a model $\langle\mathcal{I}, \epsilon\rangle$ of $S$, with $\mathbf{I}=\left(\left\langle\Delta_{i},{ }^{\mathcal{I}_{i}}\right\rangle\right)_{i \in \mathbf{O}}$ and $\epsilon=\left(\epsilon_{i}: \Delta_{i} \rightarrow \Delta_{\epsilon}\right)_{i \in \mathbf{O}}$. We must show that there exist a global configuration $\Omega$ and a local configuration $\Omega^{i}$ for each $i \in \mathbf{O}$, for which $\widehat{\mathbf{A}}_{\Omega}$ and $\left\{\widehat{O}_{\Omega^{i}}\right\}$ are consistent.

The proof is organised as follows:

1. Build the configurations according to the model $\langle\mathcal{I}, \epsilon\rangle$ :

(a) build the global configuration $\Omega$;

(b) build the local configuration $\Omega^{i}$ for each $i \in \mathbf{O}$;

(c) prove that $\Omega^{i}$ is indeed a local configuration.

2. Prove that $\widehat{\mathbf{A}}_{\Omega}$ is consistent:

(a) build an interpretation $\mathcal{I}_{A}$ of $\widehat{\mathbf{A}}_{\Omega}$;

(b) show that $\mathcal{I}_{A}=\widehat{\mathbf{A}}_{\Omega}$.

3. Prove that $\left\{\widehat{O}_{\Omega^{i}}\right\}$ is consistent for all $i \in \mathbf{O}$ :

(a) build an interpretation $\mathcal{I}_{i}$ of $\widehat{O}_{\Omega^{i}}$;

(b) show that $\mathcal{I}_{i}=\widehat{O}_{\Omega^{i}}$.

Before proceeding with the actual proof, we first define two auxiliary functions which will improve readability. Function $\varphi$ maps a concept of the global vocabulary $\mathscr{C}$ to a subset of the global domain $\Delta_{\epsilon}$.

$$
\begin{aligned}
& \varphi: \mathscr{C} \longrightarrow 2_{\epsilon}^{\Delta} \\
& i: C \longmapsto \epsilon_{i}\left(\mathcal{I}_{i}(C)\right)
\end{aligned}
$$

Function $\bar{\varphi}$ maps a concept $C$ of $\mathscr{C}$ to the complement of $\varphi(C)$ in $\Delta_{\epsilon}$.

\subsubsection{Building configurations.}

The configurations are built in the following order: global configuration and local configurations. Since local configurations have to satisfy an additional constraint, their construction is immediately followed by a proof of the validity of the definition.

Global configuration. For all $W \subseteq \mathscr{C}, W \in \Omega$ if and only if $\varphi\left(C_{W}\right) \neq \emptyset$.

Local configurations. For all $w \in \mathscr{C}_{i}, w \in \Omega^{i}$ if and only if $\mathcal{I}_{i}\left(C_{w}^{\mathscr{C}_{i}}\right) \neq \emptyset$.

Lemma $2 \Omega^{i}$ is a local configuration with respect to $\Omega$. 
Proof: Let assume that $w \in \Omega^{i}$. It implies that $\bigcap_{X \in \widehat{w}} \varphi(X) \neq \emptyset$, according to the following chain of implications:

$$
\begin{aligned}
& w \in \Omega^{i} \\
\Longrightarrow & \mathcal{I}_{i}\left(C_{w}^{\mathscr{C}_{i}}\right) \neq \emptyset \\
\Longrightarrow & \bigcap_{X \in w} \mathcal{I}_{i}(X) \neq \emptyset \\
\Longrightarrow & \epsilon_{i}\left(\bigcap_{X \in w} \mathcal{I}_{i}(X)\right) \neq \emptyset \\
\Longrightarrow & \bigcap_{X \in w} \epsilon_{i}\left(\mathcal{I}_{i}(X)\right) \neq \emptyset \\
\Longrightarrow & \bigcap_{X \in \widehat{w}} \varphi(X) \neq \emptyset
\end{aligned}
$$

There exists an element $e$ in $\bigcap_{X \in \widehat{w}} \varphi(X)$. Let us name $W$ the set $\widehat{W} \cup\{X \in \mathscr{C} \backslash \widehat{w} \mid e \in \varphi(X)\}$. By definition, $\widehat{w} \subseteq W$ and $e \in \varphi\left(C_{W}\right)$. According to the construction of $\Omega$, this ensures that $W \in \Omega$ which establishes the proof.

\subsubsection{Consistency of $\widehat{\mathbf{A}}_{\Omega}$.}

In order to prove the consistency of $\widehat{\mathbf{A}}_{\Omega}$, we first build an interpretation of the alignment ontology. There are two important properties which determine the construction of this interpretation: 1) the global interpretations of the non-empty concepts $C_{W}$ form a partition of the global domain of interpretation. More formally, $\left\{\varphi\left(C_{W}\right) \mid W \subseteq \mathscr{C} \wedge \varphi\left(C_{W}\right) \neq \emptyset\right\}$ is a partition of $\left.\Delta_{\epsilon} ; 2\right)$ because of the particularities of the axioms in $\widehat{\mathbf{A}}_{\Omega}$, it is impossible to differentiate two elements in the same set $\varphi\left(C_{W}\right)$. Therefore, it is enough to have as many elements in the domain of interpretation as there are non-empty concepts $C_{W}$. As a result, the domain of interpretation can be any set of the size of $\Omega$, for instance $\Omega$ itself.

Construction of a model of the alignment ontology. $\mathrm{A}$ model $\left\langle\Delta_{A}, .^{\mathcal{I}_{A}}\right\rangle$ for $\widehat{\mathbf{A}}_{\Omega} \operatorname{can}$ be defined as follows.

1. $\Delta_{A}=\Omega$;

2. For all $X \in \mathscr{C}$ we define $\mathcal{I}_{A}(X)=\{W \in \Omega \mid X \in W\}$;

3. For all $a_{W}$ added by Def. 11 to $\widehat{\mathbf{A}}_{\Omega}$ with assertion $C_{W} \equiv\left\{a_{W}\right\}$, we define $\mathcal{I}_{A}\left(a_{W}\right)=W$.

In the following proof, the set $\Omega$ is used for two different purposes, which might be somehow confusing in case of careless reading. Indeed, $\Omega$ represents both the domain of interpretation of the alignment ontology, and the global configuration of $S$ (i.e., a set of sets of concepts). 
Checking satisfaction of axioms. Axioms in $\widehat{\mathbf{A}}_{\Omega}$ are all introduced by the three items of Def. 11. We follow the same order to prove $\mathcal{I}_{A}$ satisfy them.

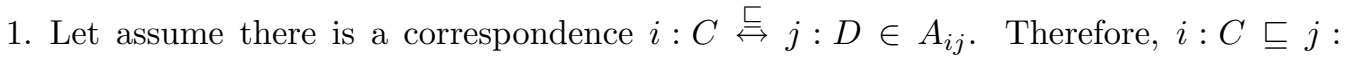
$D \in \widehat{\mathbf{A}}_{\Omega}$ is an axiom in $\widehat{\mathbf{A}}_{\Omega}$. Let $W \in \mathcal{I}_{A}(i: C)$. By construction of $\mathcal{I}_{A}, W \in \Omega$ and $i: C \in W$. If we assume that $i: D \notin W$, then $\varphi(i: C) \cap \bar{\varphi}(i: D) \neq \emptyset$ (according to the construction of $\Omega)$. So $\epsilon_{i}\left(\mathcal{I}_{i}(C)\right) \cap \Delta_{\epsilon} \backslash \epsilon_{i}\left(\mathcal{I}_{i}(D)\right) \neq \emptyset$. This contradicts the fact

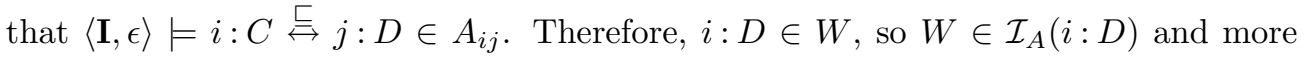
generally, $\mathcal{I}_{A}(i: C) \subseteq \mathcal{I}_{A}(i: D)$. Similarly, if $i: C \stackrel{\perp}{\Leftrightarrow} j: D$ is a correspondence in $A_{i j}$, then $i: C \sqsubseteq \neg j: D \in \widehat{\mathbf{A}}_{\Omega}$. Let $W \in \mathcal{I}_{A}(i: C)$. If we assume that $i: D \in W$, then $\varphi(i: C) \cap \varphi(i: D) \neq \emptyset$ (according to the construction of $\Omega$ ). So $\epsilon_{i}\left(\mathcal{I}_{i}(C)\right) \cap \epsilon_{i}\left(\mathcal{I}_{i}(D)\right) \neq \emptyset$. This contradicts the fact that $\langle\mathbf{I}, \epsilon\rangle \models i: C \stackrel{\perp}{\Leftrightarrow} j: D \in A_{i j}$. Therefore, $i: D \notin W$ and more generally, $\mathcal{I}_{A}(i: C) \subseteq \Omega \backslash \mathcal{I}_{A}(i: D)$.

2. If $W \in \Omega$, then $C_{W} \equiv\left\{a_{W}\right\} \in \widehat{\mathbf{A}}_{\Omega}$. By construction of $\mathcal{I}_{A}$,

$$
\begin{aligned}
\mathcal{I}_{A}\left(C_{W}\right) & =\bigcap_{X \in W} \mathcal{I}_{A}(X) \cap \bigcap_{X \in \mathscr{C} \backslash W} \Omega \backslash \mathcal{I}_{A}(X) \\
& =\bigcap_{X \in W}\{\omega \in \Omega \mid X \in \omega\} \cap \bigcap_{X \in \mathscr{C} \backslash W} \Omega \backslash\{\omega \in \Omega \mid X \in \omega\}
\end{aligned}
$$

Quite obviously, for all $X \in W, W \in\{\omega \in \Omega \mid X \in \omega\}$. Moreover, for all $X \in \mathscr{C} \backslash W$, $W \notin\{\omega \in \Omega \mid X \in \omega\}$. Therefore $\mathcal{I}_{A}\left(a_{W}\right)=W \in \mathcal{I}_{A}\left(C_{W}\right)$. Now, let $W^{\prime} \in \mathcal{I}_{A}\left(C_{W}\right)$. According to $(*)$, it is obvious that for all $X \in W, X \in W^{\prime}$ and for all $X \in \mathscr{C} \backslash W$, $X \notin W^{\prime}$. As a result, $W=W^{\prime}$. From this and $\mathcal{I}_{A}\left(a_{W}\right)=W \in \mathcal{I}_{A}\left(C_{W}\right)$, we conclude that $\left\{\mathcal{I}_{A}\left(a_{W}\right)\right\}=\mathcal{I}_{A}\left(C_{W}\right)$.

3. If $W \in \mathscr{C} \backslash \Omega$, then $C_{W} \sqsubseteq \perp \in \widehat{\mathbf{A}}_{\Omega}$, which means that $\varphi\left(C_{W}\right)=\emptyset$ by construction of $\Omega$. The proof of the previous item established that if $W^{\prime} \in \mathcal{I}_{A}\left(C_{W}\right)$ then necessarily, $W^{\prime}=W$. But it cannot be so here because $\mathcal{I}_{A} \subseteq \Omega$ and $W \notin \Omega$. So $\mathcal{I}_{A}\left(C_{W}\right)=\emptyset$.

So $\mathcal{I}_{A} \models \widehat{\mathbf{A}}_{\Omega}$.

\subsubsection{Consistency of $\widehat{O}_{\Omega^{i}}$.}

In order to build a valid interpretation of $\widehat{O}_{\Omega^{i}}$, we first establish a preliminary result.

Lemma 3 If $W \in \Omega$ and $\left.X \in W\right|_{i}$, then there exists $e \in \mathcal{I}_{i}(X) \cap \bigcap_{\left.X^{\prime} \in \mathscr{C}_{i} \backslash W\right|_{i}} \Delta_{i} \backslash \mathcal{I}_{i}\left(X^{\prime}\right)$ such that $\epsilon_{i}(e) \in \varphi\left(C_{W}\right)$.

Proof: Let us assume that $W \in \Omega$ and $\left.X \in W\right|_{i}$. Then, by construction of $\Omega, \varphi\left(C_{W}\right) \neq \emptyset$. So there exists $x \in \varphi\left(C_{W}\right)$. It follows that $x \in \varphi(i: X) \cap \bigcap_{\left.X^{\prime} \in \mathscr{C}_{i} \backslash W\right|_{i}} \bar{\varphi}\left(i: X^{\prime}\right)$. So there 
exists $e \in \mathcal{I}_{i}(X)$ such that $\epsilon_{i}(e)=x$. Moreover, for all $\left.X^{\prime} \in \mathscr{C}_{i} \backslash W\right|_{i}, \epsilon_{i}(e) \in \bar{\varphi}\left(i: X^{\prime}\right)$, so $e \notin \mathcal{I}_{i}\left(X^{\prime}\right)$. As a result, $e \in \mathcal{I}_{i}(X) \cap \bigcap_{\left.X^{\prime} \in \mathscr{C}_{i} \backslash W\right|_{i}} \Delta_{i} \backslash \mathcal{I}_{i}\left(X^{\prime}\right)$ and $\epsilon_{i}(e) \in \varphi\left(C_{W}\right)$, which establishes the proof.

Construction of a model of the extended ontology. We now devise a model $\left\langle\Delta_{i}^{\prime},{ }^{\mathcal{I}^{\prime}}\right\rangle$ for each $\widehat{O}_{\Omega^{i}}$.

1. $\Delta_{i}^{\prime}:=\Delta_{i}$;

2. $\mathcal{I}_{i}^{\prime}(C):=\mathcal{I}_{i}(C)$ for all concept name $C$ in $O_{i}$, and thus, in $\widehat{O}_{\Omega^{i}}$;

3. $\mathcal{I}_{i}^{\prime}(R):=\mathcal{I}_{i}(R)$ for all role name $R$ in $O_{i}$, and thus, in $\widehat{O}_{\Omega^{i}}$;

4. $\mathcal{I}_{i}^{\prime}(o):=\mathcal{I}_{i}(o)$ for all individual name $o$ in $O_{i}$, and thus, in $\widehat{O}_{\Omega^{i}}$;

5. for all individual $b_{w}$ added by Def. 13 to $\widehat{O}_{\Omega^{i}}$ with assertion $C_{w}^{\mathscr{C}_{i}}\left(b_{w}\right)$, we define $\mathcal{I}_{i}\left(b_{w}\right)=$ $x$ for any $x \in \mathcal{I}_{i}\left(C_{w}^{\mathscr{C}_{i}}\right.$ (which exists, according to the construction of $\Omega^{i}$ );

6. for all concept $C_{W}^{X}$ added by Def. 13 to $\widehat{O}_{\Omega^{i}}$, we define $\mathcal{I}_{i}\left(C_{W}^{X}\right)=\left\{e \in \mathcal{I}_{i}(X) \cap\right.$ $\left.\bigcap_{\left.X^{\prime} \in \mathscr{C}_{i} \backslash W\right|_{i}} \Delta_{i} \backslash \mathcal{I}_{i}(X) \mid \epsilon_{i}(e) \in \varphi\left(C_{W}\right)\right\}$.

7. for all individual $b_{W}^{X}$ added by Def. 13 to $\widehat{O}_{\Omega^{i}}$ with assertion $C_{W}^{X}\left(b_{W}^{X}\right)$, we define $\mathcal{I}_{i}\left(b_{W}^{X}\right)=x$ for any $x \in \mathcal{I}_{i}^{\prime}\left(C_{W}^{X}\right)$ (which exists according to Lem. 3 ).

Checking satisfaction of axioms. Axioms existing in ontology $O_{i}$ are obviously satisfied by $\mathcal{I}_{i}^{\prime}$. Here, we demonstrate that the new axioms brought by $\Omega^{i}$ are satisfied, then prove that the axioms brought by the global configuration are also satisfied.

1. Satisfaction of $C_{w}^{\mathscr{C}_{i}}\left(b_{w}\right)$. Let $w \in \Omega^{i}$. By construction of $\mathcal{I}_{i}^{\prime}, \mathcal{I}_{i}^{\prime}\left(b_{w}\right) \in \mathcal{I}_{i}\left(C_{w}^{\mathscr{C}}\right)$. So $\mathcal{I}_{i}^{\prime}=C_{w}^{\mathscr{C}_{i}}\left(b_{w}\right)$.

2. Satisfaction of $C_{w}^{\mathscr{C}_{i}} \sqsubseteq \perp$. Let $w \in \mathscr{C}_{i} \backslash \Omega^{i}$. By construction of $\Omega^{i}, \mathcal{I}_{i}\left(C_{w}^{\mathscr{C}_{i}}\right)=\emptyset$. So $\mathcal{I}_{i}^{\prime}=C_{w}^{\mathscr{C}_{i}} \sqsubseteq \perp$.

3. Satisfaction of $C_{W}^{X} \sqsubseteq X \sqcap \prod_{\left.X^{\prime} \in \mathscr{C}_{i} \backslash W\right|_{i}} X^{\prime}$. This is trivial according to the construction of $\mathcal{I}_{i}^{\prime}$.

4. Satisfaction of $C_{W}^{X}\left(b_{W}^{X}\right)$. This is obvious thanks to Lem. 3 and the construction of $\mathcal{I}_{i}^{\prime}$.

5. Satisfaction of $C_{W}^{X} \sqsubseteq C_{W}^{\top}$. The definition of $\mathcal{I}_{i}^{\prime}$ trivially implies that $\mathcal{I}_{i}^{\prime}\left(C_{W}^{X}\right) \sqsubseteq$ $\mathcal{I}_{i}^{\prime}\left(C_{W}^{\top}\right)$. 
6. Satisfaction of $C_{W}^{\top} \sqsubseteq \neg C_{W^{\prime}}^{\top}$. For all $e \in \mathcal{I}_{i}^{\prime}\left(C_{W}^{\top}\right), \epsilon_{i}(e) \in \varphi\left(C_{W}\right)$ and for all $f \in$ $\mathcal{I}_{i}^{\prime}\left(C_{W^{\prime}}^{\top}\right), \epsilon_{i}(f) \in \varphi\left(C_{W^{\prime}}\right)$. But $\varphi\left(C_{W}\right)$ and $\varphi\left(C_{W^{\prime}}\right)$ are disjoint sets, so $\epsilon_{i}(e) \neq \epsilon_{i}(f)$. Therefore $e \neq f$, and more generally, $\mathcal{I}_{i}^{\prime}\left(C_{W}^{\top}\right) \cap \mathcal{I}_{i}^{\prime}\left(C_{W^{\prime}}^{\top}\right)$.

We have defined functions $\mathcal{I}_{i}^{\prime}$ and showed that $\mathcal{I}_{i}^{\prime}$ is a model for $\widehat{O}_{\Omega^{i}}$ for all $i$.

\section{An Algorithm for Consistency Checking}

From the construction of alignment and extended ontologies introduced in Section 5 , we can devise an algorithm (Alg. 7) for reasoning on a IDDL system $S$. The algorithm performs searching valid configurations from which a consistent alignment ontology and extended ontologies can be built. If the algorithm can not find any such configuration then $S$ is inconsistent. The correction of the algorithm is ensured by Theorem 1 .

Property 1 Given $c$ the number of global concepts in $\mathscr{C}$ and $N$ the number of ontologies in the system, the complexity of Alg. 7 is double exponential and bounded by $N 2^{\left(2^{c+1}\right)}$ (the number of call to local consistency checking).

Proof: There are as many global configurations as there are subsets of $\mathscr{2}^{\mathscr{C}}$, i.e., $2^{\left(2^{c}\right)}$. For each global configuration, all local configurations have to be tested, for all ontologies. The number of local configuration, for a given ontology in the system, is bounded by $2^{\left(2^{c}\right)}$, and there are $N$ ontologies to be checked. So the total number of consistency checking is bounded by $N 2^{\left(2^{c}\right)} \cdot 2^{\left(2^{c}\right)}=N 2^{\left(2^{c+1}\right)}$.

Thus, the complexity of Alg. 7 is bounded by a double exponential function in the size of alignments. However, when we look at the way of the construction of global configurations (which is responsible for the double exponential blow-up), the number of global configurations which are necessarily considered in the algorithm can be dramatically reduced by pre-verifying if $\mathbf{A}=\Omega_{W}$ or $O_{i} \models \Omega_{W}$ with a local reasoner. Additionally, a smart implementation with help of a cache mechanisms would improve considerably local reasoning. Such an optimization would take advantage of the fact that if two extended ontologies are built from one local ontology with two local configurations then their structures of models (if they exist) are slightly different from each other.

The following examples illustrate Alg. 7

Example 2 By using Alg. 7, we show that the IDDL system introduced in Example 1 is consistent.

Example 3 Let $O_{1}=\left\{C_{1} \equiv A_{1} \sqcap B_{1}\right\}, O_{2}=\left\{A_{2} \sqsubseteq \top, B_{2}(a)\right\}$ and alignment $A_{12}=$ $\left\{A_{1} \perp B_{1}, C_{1} \sqsupseteq B_{2}\right\}$.

- In order to ensure that the alignment ontology $\widehat{A}$ is consistent the components of global configurations including both $A_{1}, B_{1}$ or both $C_{1}, \neg B_{2}$ simultaneously must be unsatisfiable. So the following components are possibly non-unsatisfiable: $\Omega_{\left\{A_{1}\right\}}, \Omega_{\left\{B_{1}\right\}}$, 


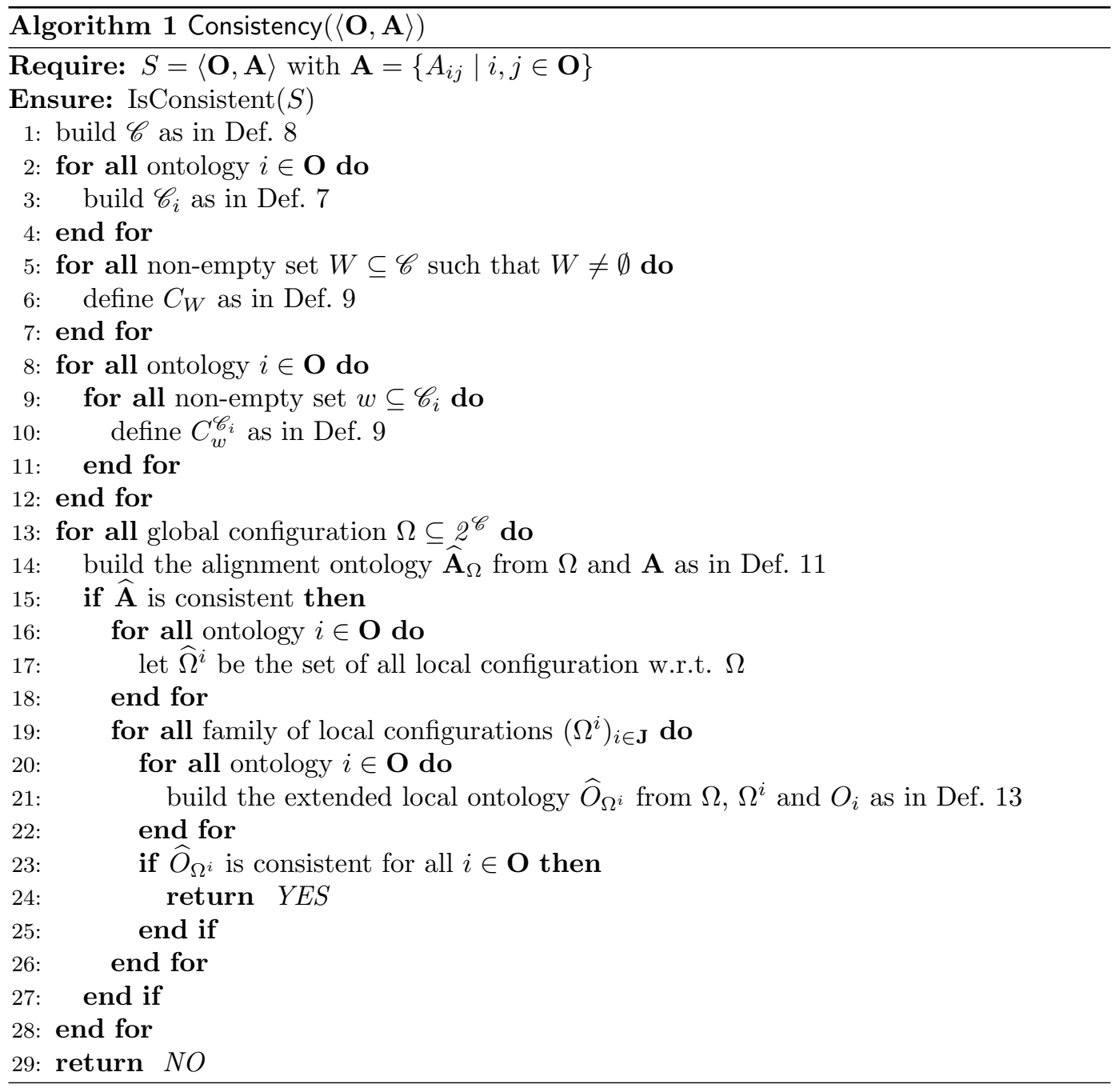

$\Omega_{\left\{A_{1}, B_{2}, C_{1}\right\}}, \Omega_{\left\{B_{1}, B_{2}, C_{1}\right\}}, \Omega_{\left\{B_{2}, C_{1}\right\}}$ and $\Omega_{\left\{A_{1}, B_{1}, B_{2}, C_{1}\right\}}$ (note that omitting concepts $\mathrm{T}_{1}, \mathrm{~T}_{2}$ from configurations does not change anything about the reasoning for this example).

- To ensure the consistency of $\widehat{A}$, it is not possible that $\Omega_{\left\{A_{1}, B_{1}, B_{2}, C_{1}\right\}}$ is non-unsatisfiable due to the disjointness between $A_{1}$ and $B_{1}$ in $\widehat{A}$. Moreover, in order to guarantee the consistency of $\widehat{O}_{1}$ it is not possible that three components $\Omega_{\left\{A_{1}, B_{2}, C_{1}\right\}}, \Omega_{\left\{B_{1}, B_{2}, C_{1}\right\}}$, 
$\Omega_{\left\{B_{2}, C_{1}\right\}}$ are non-unsatisfiable since if $C_{1}$ is non-unsatisfiable then $A_{1}$ and $B_{1}$ are so. Thus, it remains $\Omega_{\left\{A_{1}\right\}}, \Omega_{\left\{B_{1}\right\}}$ which are possibly non-unsatisfiable.

- On the other hand, in order to guarantee the consistency of $\widehat{O}_{2}$ there exists at least a component of global configuration containing $B_{2}$ which is non-unsatisfiable. This is due to the non-unsatisfiability of $\mathrm{B}_{2}$ in $\widehat{\mathrm{O}}_{2}$. However, the two remaining possibly non-unsatisfiable components do not include $B_{2}$.

According to Alg. 7 , the system $S$ is not consistent.

Although the algorithm does not involve a tableaux-like process, there is a strong connection between the construction of global configurations in Alg. 7 and the construction of a tableau for concept satisfiability. In fact, a clash-free tableau for alignment ontology corresponds to a global configuration such that the alignment ontology is consistent. Instead of selecting appropriate subsets of labels by applying expansion rules to each node with a set of labels, Alg. 7 considers all possible combinations of subsets of labels. This exhaustive behaviour is required in the distributed reasoning context where it is needed to know all clash-free tableaux in the worst case. This can be seen as a drawback in terms of algorithmic complexity, but is also an advantage in terms of versatility. So far, no other distributed formalism is able to treat a network of $\mathcal{S R O I Q}$ ontologies.

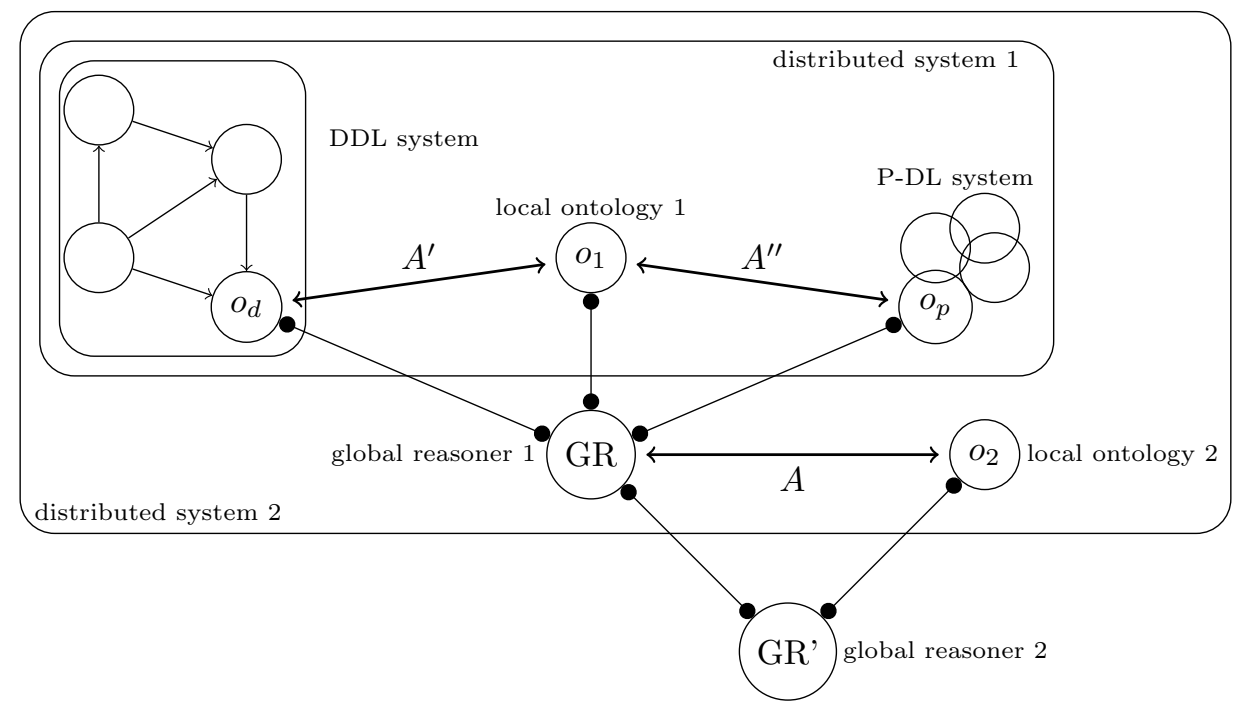

Figure 4: Hybrid distributed system. Local ontologies are associated with local reasoners.

Another advantage of the algorithm resides in the encapsulation of local reasoners. The algorithm asks to local reasoners for the consistency of an extended ontology, which is simply 
the local ontology extended with axioms that are in the language of most description logic based reasoners. In fact, local reasoners can themselves be peers of a distributed system, as shown in Fig. 4. In fact, any formalism can be used at the local level as soon as there exists a reasoner which can check consistency of its internal ontology extended with the axioms introduced by the alignments.

\section{Reasoning with Cross-ontology Role Subsumption}

In this section, we devise a new reasoning procedure which now takes into account crossontology role subsumption. The principle behind this improved reasoning task is the same as before, except that configurations must be extended to take into account the roles involved in correspondences. Since most of the definitions necessary for this part are the same or similar as the ones for the previous part, we simply update existing definitions or add new definitions when necessary.

First, a new notion of role vocabulary must be defined, locally or globally.

Definition 14 (Local role vocabulary) Let $S=\langle\mathbf{O}, \mathbf{A}\rangle$ be a DS. We denote by $\mathscr{R}_{i}$ the set that includes primitive or complex roles that appear in the left side of correspondences in $A_{i j}$ or in the right side of correspondences in $A_{j i}$ together with their inverse roles (i.e., $\left.R \in \mathscr{R}_{i} \Leftrightarrow R^{-} \in \mathscr{R}_{i}\right)$.

Definition 15 (Global role vocabulary) Let $S=\langle\mathbf{O}, \mathbf{A}\rangle$ be a DS. The set of global global role names of $S$ is $\mathscr{R}=\bigcup_{i \in \mathbf{O}}\left\{i: R \mid R \in \mathscr{R}_{i}\right\}$.

Now we must define a new kind of configuration which has to be considered in addition to the already defined global and local configurations. However, the treatment of roles is quite different from the treatment of concepts only, because there are interactions between roles and concepts. Therefore, we need to keep track of role satisfiability in addition to concept satisfiability.

In fact, we do that by considering a given (concept) configuration $\Omega$ which represents a partition of the domain of interpretation. Then, according to this configuration, we define the role configuration as a family of relations over $\Omega$ indexed by the set of roles. In other terms, we determine in a role configuration whether there exists a relation $R$ between two sets in the partition $\Omega$.

Definition 16 (Role configuration) Let $S$ be a DS with a set of global role names $\mathscr{R}$. Let $\Omega$ be a global configuration of $S$. A role configuration of $S$ with respect to $\Omega$ is a subset $\Phi_{\Omega}$ of $\Omega \times \Omega \times \mathscr{R}$.

The introduction of role configuration leads to additional constraints on the alignment ontology that we summarize in this addendum to Def. 11 . 
Definition 17 (Alignment ontology (revised)) Let $S=\langle\mathbf{O}, \mathbf{A}\rangle$ be a DS. Let $\Omega$ be a global configuration of $S$, and let $\Phi_{\Omega}$ be a role configuration with respect to $\Omega$. The alignment ontology with respect to $\Omega$ and $\Phi_{\Omega}$ is an ontology $\widehat{\mathbf{A}}_{\Omega}$ defined as follows:

1. 2. and 3. See Def. 11:

4. for each $i, j \in \mathbf{O}$, if $i: R \stackrel{\sqsubseteq}{\leftrightarrows}: S$ is a role correspondence in $\mathbf{A}$ then $i: R \sqsubseteq j: S$ is a sub-role axiom of $\widehat{\mathbf{A}}_{\Omega}$;

5. for each $\left\langle W, W^{\prime}, R\right\rangle \in \Phi_{\Omega},\left\{a_{W}\right\} \sqsubseteq \exists R .\left\{a_{W^{\prime}}\right\}$ is an axiom of $\widehat{\mathbf{A}}_{\Omega}$;

6. for each $\left\langle W, W^{\prime}, R\right\rangle \notin \Phi_{\Omega},\left\{a_{W}\right\} \sqsubseteq \forall R . \neg\left\{a_{W^{\prime}}\right\}$ is an axiom of $\widehat{\mathbf{A}}_{\Omega}$;

Similarly to local configuration, local role configurations have to satisfy a certain constraint imposed by the global configuration.

Definition 18 (Local role configuration) Let $S$ be a DS with a set of global role names $\mathscr{R}$. Let $\Omega$ be a global configuration of $S$, let $\Phi_{\Omega}$ be a role configuration with respect to $\Omega$, and $\Omega^{i}$ a local configuration of $O_{i}$ with respect to $\Omega$. A local role configuration of $O_{i}$ with respect to $\Omega, \Omega_{i}$ and $\Phi_{\Omega}$ is a subset $\Phi_{\Omega^{i}}$ of $\Omega^{i} \times \Omega^{i} \times \mathscr{R}_{i}$ such that $\left\langle w, w^{\prime}, R\right\rangle \in \Phi_{\Omega^{i}}$ implies that there exists $W, W^{\prime} \in \Omega$ such that $\left.w \subseteq W\right|_{i},\left.w^{\prime} \subseteq W^{\prime}\right|_{i}$ and $\left\langle W, W^{\prime}, i: R\right\rangle \in \Phi_{\Omega}$.

The extended ontologies are now further extended with axioms which involve roles.

Definition 19 (Extended ontologies (revised)) Let $S=\langle\mathbf{O}, \mathbf{A}\rangle$ be a DS. Let $\Omega$ be a global configuration and $\Phi_{\Omega}$ a role configuration of $S$. For each $O_{i} \in \mathbf{O}$, let $\Omega^{i}$ be a local configuration with respect to $\Omega$ and $\Phi_{\Omega^{i}}$ be a local role configuration with respect to $\Omega, \Omega^{i}$ and $\Phi_{\Omega}$. The extended ontology $\widehat{O}_{\Omega^{i}}$ with respect to $\Omega^{i}, \Omega, \Phi_{\Omega}$ and $\Phi_{\Omega^{i}}$ is defined as follows:

1. 2. 3. 4. and 5. See Def. 13:

6. for each $\left\langle w, w^{\prime}, R\right\rangle \in \Phi_{\Omega^{i}},\left(C_{w}^{\mathscr{C}_{i}} \sqcap \exists R . C_{w^{\prime}}^{\mathscr{C}_{i}}\right)\left(b_{w, w^{\prime}}^{R}\right)$ is an axiom of $\widehat{O}_{\Omega^{i}}$, where $b_{w, w^{\prime}}^{R}$ is a new individual name;

7. for each $\left\langle w, w^{\prime}, R\right\rangle \notin \Phi_{\Omega^{i}}, C_{w}^{\mathscr{C}_{i}} \sqsubseteq \forall R . \neg C_{w^{\prime}}^{\mathscr{C}_{i}}$ is an axiom of $\widehat{O}_{\Omega^{i}}$;

8. for each $W, W^{\prime} \subseteq \Omega$, and each $R \in \mathscr{R}_{i}$, we define a new concept name $C_{W, W^{\prime}}^{R}$ for ontology $\widehat{O}_{\Omega^{i}}$ such that:

(a) $C_{W, W^{\prime}}^{R} \sqsubseteq C_{W}^{\top}$ is an axiom of $\widehat{O}_{\Omega^{i}}$;

(b) if $\left\langle W, W^{\prime}, i: R\right\rangle \in \Phi_{\Omega}$ then $C_{W, W^{\prime}}^{R} \sqsubseteq \exists R . C_{W^{\prime}, W}^{R^{-}}$and $C_{W, W^{\prime}}^{R}\left(\beta_{W, W^{\prime}}^{R}\right)$ are axioms of $\widehat{O}_{\Omega^{i}}$ with $\beta_{W, W^{\prime}}^{R}$ a new individual name;

(c) else, $C_{W, W^{\prime}}^{R} \sqsubseteq \forall R . \neg C_{W^{\prime}, W}^{R^{-}}$is an axiom of $\widehat{O}_{\Omega^{i}}$; 
9. for each $R \in \mathscr{R}_{i}, \exists R . \top \sqsubseteq \bigsqcup_{W, W^{\prime} \in \Omega} C_{W, W^{\prime}}^{R}$ is an axiom of $\widehat{O}_{\Omega^{i}}$.

In the previous definition, item 6 means that a triple $\left\langle w, w^{\prime}, R\right\rangle$ in the local role configuration determines the existence of a relation $R$ between some member of $C_{w}^{\mathscr{C}_{i}}$ and some member of $C_{w^{\prime}}^{\mathscr{C}_{i}}$. Conversely, item 7 means that whenever a triple $\left\langle w, w^{\prime}, R\right\rangle$ is not in the local role configuration, then concepts $C_{w}^{\mathscr{C}_{i}}$ and $C_{w^{\prime}}^{\mathscr{C}_{i}}$ are not related through $R$. Item 8 adds a concept $C_{W, W}^{R}$ which represents the set of elements of local concept $C_{W}^{\top}$ which have their couterparts in global concept $C_{W}$ and are in relation through $R$ with elements which have their own counterparts in $C_{W^{\prime}}$. Finally, item 9 asserts that any element involve in a relation $R$ must belong to one of the newly introduced sets $C_{W, W^{\prime}}^{R}$ for some $W$ and $W^{\prime}$. This last item is important to ensure that the role structure is correctly propagated.

Theorem 2 (DS Consistency) Let $S=\langle\mathbf{O}, \mathbf{A}\rangle$ be a DS. $S$ is consistent if and only if there exist a global configuration $\Omega$ of $S$, a role configuration $\Phi_{\Omega}$ with respect to $\Omega$, local configurations $\Omega^{i}$ for all $O_{i} \in \mathbf{O}$ with respect to $\Omega$ and local role configurations $\Phi_{\Omega^{i}}$ with respect to $\Omega, \Omega^{i}$ and $\Phi_{\Omega}$, such that the alignment ontology $\widehat{\mathbf{A}}_{\Omega}$ and the extended local ontologies $\left\{\widehat{O}_{\Omega^{i}}\right\}$ as defined in Def. 17 and Def. 19 are consistent.

Proof: The proof of the new version of the theorem has a lot in common with the previous version, so we only present in this section the modifications or additions necessary to deal with role correspondences. Moreover, the outline of this proof is the same as the previous one.

\subsection{If-direction $\Leftarrow$}

In this section, the only change is in the additional role correspondence satisfaction. Other definitions are kept identical, in particular the equalizing function is defined as before. However, in order to demonstrate the satisfaction of cross-ontology role subsumptions, we introduce a new lemma, which can be considered the counterpart of Lem. 1 for roles.

\subsubsection{Auxiliary lemmas.}

Informally, we could be stated as follows: the function $\epsilon_{i}$ built before preserves the structure of roles.

Lemma 4 For each $R \in \mathscr{R}_{i}, \epsilon_{i}\left(\mathcal{I}_{i}(R)\right)=\left\{\left\langle\mathcal{I}_{A}\left(a_{W}\right), \mathcal{I}_{A}\left(a_{W^{\prime}}\right)\right\rangle \mid\left\langle W, W^{\prime}, i: R\right\rangle \in \Phi_{\Omega}\right\}$.

Proof: The proof of this lemma is decomposed into two steps, corresponding to both inclusions of the two sets. 
$(\subseteq)$ : Let $\left(x, x^{\prime}\right) \in \epsilon_{i}\left(\mathcal{I}_{i}(R)\right)$. There exists $\left(e, e^{\prime}\right) \in \mathcal{I}_{i}(R)$ such that $x=\epsilon_{i}(e)$ and $x^{\prime}=$ $\epsilon_{i}\left(e^{\prime}\right)$. It follows that $e \in \mathcal{I}_{i}(\exists R$. $\top)$. So, thanks to item 9 of Def. 19 , and because $\mathcal{I}_{i}$ is a model of $\widehat{O}_{\Omega^{i}}$, there exists $W, W^{\prime} \in \Omega$ such that $e \in \mathcal{I}_{i}\left(C_{W, W^{\prime}}^{R}\right)$ and similarly, $e^{\prime} \in \mathcal{I}_{i}\left(C_{W^{\prime}, W}^{R^{-}}\right)$. This implies that $\widehat{O}_{\Omega^{i}} \not \models C_{W, W^{\prime}}^{R} \sqsubseteq \forall R \neg C_{W^{\prime}, W}^{R-}$, which means that $\left\langle W, W^{\prime}, R\right\rangle \in \Phi_{\Omega}$. Additionally, thanks to item 8a of Def. 19, $e \in \mathcal{I}_{i}\left(C_{W}^{\top}\right)$ and $e^{\prime} \in \mathcal{I}_{i}\left(C_{W^{\prime}}^{\top}\right)$, so $\epsilon_{i}(e)=\mathcal{I}_{A}\left(a_{W}\right)$ and $\epsilon_{i}\left(e^{\prime}\right)=\mathcal{I}_{A}\left(a_{W^{\prime}}\right)$. This establishes the first inclusion.

( $\supseteq$ ): $\quad$ Let $\left\langle W, W^{\prime}, i: R\right\rangle \in \Phi_{\Omega}$. According to item $8 \mathrm{~b}$ of Def. 19, we have $C_{W, W^{\prime}}^{R}\left(\beta_{W, W^{\prime}}^{R}\right)$ and $C_{W, W^{\prime}}^{R} \sqsubseteq \exists R . C_{W^{\prime}, W}^{R^{-}}$, which implies that there exists $x \in \mathcal{I}_{i}\left(C_{W^{\prime}, W}^{R^{-}}\right)$such that $\left\langle\mathcal{I}_{i}\left(\beta_{W, W^{\prime}}^{R}\right), x\right\rangle \in$ $\mathcal{I}_{i}(R)$. Moreover, by item 8 a of Def. 19 we have $\mathcal{I}_{i}\left(\beta_{W, W^{\prime}}^{R}\right) \in \mathcal{I}_{i}\left(C_{W}^{\top}\right)$ and $x \in \mathcal{I}_{i}\left(C_{W^{\prime}}^{\top}\right)$, so by definition of $\epsilon_{i}, \epsilon_{i}\left(\mathcal{I}_{i}\left(\beta_{W, W^{\prime}}^{R}\right)\right)=\mathcal{I}_{A}\left(a_{W}\right)$ and $\epsilon_{i}(x)=\mathcal{I}_{A}\left(a_{W^{\prime}}\right)$. Therefore, $\left\langle\mathcal{I}_{A}\left(a_{W}\right), \mathcal{I}_{A}\left(a_{W^{\prime}}\right)\right\rangle \in$ $\epsilon_{i}\left(\mathcal{I}_{i}(R)\right)$. This establishes the other inclusion.

\subsubsection{Satisfaction of the system.}

Cross-ontology role subsumption $\left(i: R \sqsubseteq j: S \in A_{i j} \Longrightarrow \epsilon_{i}\left(\mathcal{I}_{i}(R)\right) \subseteq \epsilon_{j}\left(\mathcal{I}_{j}(S)\right)\right)$. Let us assume that $i: R \stackrel{\sqsubseteq}{\Leftrightarrow} j: S \in A_{i j}$. Let $\left\langle x, x^{\prime}\right\rangle \in \epsilon_{i}\left(\mathcal{I}_{i}(R)\right)$. From Lem. 44 it follows that there exist $W, W^{\prime} \in \Omega$ such that $x=\mathcal{I}_{A}\left(a_{W}\right), x^{\prime}=\mathcal{I}_{A}\left(a_{W^{\prime}}\right)$ and $\left\langle W, W^{\prime}, i: R\right\rangle \in \Phi_{\Omega}$. By the definition of $\widehat{\mathbf{A}}_{\Omega}$, we deduce that $\left\langle\mathcal{I}_{A}\left(a_{W}\right), \mathcal{I}_{A}\left(a_{W^{\prime}}\right)\right\rangle \in \mathcal{I}_{A}(i: R)$. Moreover, due to the presence of the role subsumption $i: R \stackrel{\sqsubseteq}{\Leftrightarrow}: S$, the axiom $i: R \sqsubseteq j: S$ is satisfied by $\mathcal{I}_{A}$. Therefore $\left\langle\mathcal{I}_{A}\left(a_{W}\right), \mathcal{I}_{A}\left(a_{W^{\prime}}\right)\right\rangle \in \mathcal{I}_{A}(j: S)$ and so $\left\langle W, W^{\prime}, j: S\right\rangle \in \Phi_{\Omega}$. Hence, thanks to Lem. 4. we obtain $\left\langle\mathcal{I}_{A}\left(a_{W}\right), \mathcal{I}_{A}\left(a_{W^{\prime}}\right)\right\rangle \in \epsilon_{j}\left(\mathcal{I}_{j}(S)\right)$. Therefore $\left\langle x, x^{\prime}\right\rangle \in \epsilon_{j}\left(\mathcal{I}_{j}(S)\right)$ and more generally, $\epsilon_{i}\left(\mathcal{I}_{i}(R)\right) \subseteq \epsilon_{j}\left(\mathcal{I}_{j}(S)\right)$.

\subsection{Only-if-direction}

The new version of this part of the proof is organised as follows:

1. Build the configurations according to the model $\langle\mathcal{I}, \epsilon\rangle$ :
(a) build the global configuration $\Omega$; (done)
(b) build the role configuration $\Phi_{\Omega}$ with respect to $\Omega$;
(c) build the local configurations $\Omega^{i}$ with respect to $\Omega$; (done)
(d) prove that $\Omega^{i}$ is indeed a local configuration; (done)
(e) build the local role configurations $\Phi_{\Omega^{i}}$ with respect to $\Omega^{i}$ and $\Phi_{\Omega}$;
(f) prove that $\Phi_{\Omega^{i}}$ is indeed a local role configuration.

2. Prove that $\widehat{\mathbf{A}}_{\Omega}$ is consistent:

(a) build an interpretation $\mathcal{I}_{A}$ of $\widehat{\mathbf{A}}_{\Omega}$;

(b) show that $\mathcal{I}_{A}=\widehat{\mathbf{A}}_{\Omega}$.

3. Prove that $\left\{\widehat{O}_{\Omega^{i}}\right\}$ is consistent for all $i \in \mathbf{O}$ :

(a) build an interpretation $\mathcal{I}_{i}$ of $\widehat{O}_{\Omega^{i}}$; 
(b) show that $\mathcal{I}_{i}=\widehat{O}_{\Omega^{i}}$.

Before proceeding with this part of the proof, we first extend the function $\varphi$ to also treat the interpretation of roles.

$$
\begin{array}{rlrl}
\varphi: \mathscr{C} \cup \mathscr{R} & \longrightarrow 2_{\epsilon}^{\Delta} \cup \mathscr{2}_{\epsilon}^{\Delta} \times \Delta_{\epsilon} & \\
i: C \longmapsto \epsilon_{i}\left(\mathcal{I}_{i}(C)\right) & & \text { if } C \in \mathscr{C}_{i} \\
i: R & \longmapsto \epsilon_{i}\left(\mathcal{I}_{i}(R)\right) & & \text { if } R \in \mathscr{R}_{i}
\end{array}
$$

This will help simplifying the notations.

\subsubsection{Building configurations.}

Global and local configuration can be defined exactly as in Sect.6.2.1. The construction of global and local role configuration follows, according to the same notations as before.

Role configuration. For all $W, W^{\prime} \in \Omega$ and all $R \in \mathscr{R},\left\langle W, W^{\prime}, R\right\rangle \in \Omega$ if and only if

$$
\varphi(R) \cap\left(\varphi\left(C_{W}\right) \times \varphi\left(C_{W^{\prime}}\right)\right) \neq \emptyset
$$

Local role configurations. For all $w, w^{\prime} \in \Omega_{i}$ and all $R \in \mathscr{R}_{i},\left\langle w, w^{\prime}, R\right\rangle \in \Phi_{\Omega^{i}}$ if and only if

$$
\mathcal{I}_{i}(R) \cap\left(\mathcal{I}_{i}\left(C_{w}^{\mathscr{C}_{i}}\right) \times \mathcal{I}_{i}\left(C_{w^{\prime}}^{\mathscr{C}_{i}}\right)\right) \neq \emptyset
$$

Local role configurations have to satisfy a specific constraint, so it must be proved that the previous construction is indeed a local role configuration.

Lemma $5 \Phi_{\Omega^{i}}$ is a local role configuration with respect to $\Omega^{i}$ and $\Phi_{\Omega}$.

Proof: Let assume that $\left\langle w, w^{\prime}, R\right\rangle \in \Phi_{\Omega^{i}}$. It implies that

$$
\varphi(i: R) \cap\left(\bigcap_{X \in \widehat{w}} \varphi(X) \times \bigcap_{X^{\prime} \in \widehat{w}^{\prime}} \varphi\left(X^{\prime}\right)\right) \neq \emptyset
$$


according to the following chain of implications:

$$
\begin{aligned}
& \left\langle w, w^{\prime}, R\right\rangle \in \Phi_{\Omega^{i}} \\
\Longrightarrow & \mathcal{I}_{i}(R) \cap\left(\varphi\left(C_{W}\right) \times \varphi\left(C_{W^{\prime}}\right)\right) \neq \emptyset \\
\Longrightarrow & \mathcal{I}_{i}(R) \cap\left(\bigcap_{X \in w} \mathcal{I}_{i}(X) \times \bigcap_{X^{\prime} \in w^{\prime}} \mathcal{I}_{i}\left(X^{\prime}\right)\right) \neq \emptyset \\
\Longrightarrow & \epsilon_{i}\left(\mathcal{I}_{i}(R)\right) \cap\left(\bigcap_{X \in w} \epsilon_{i}\left(\mathcal{I}_{i}(X)\right) \times \bigcap_{X^{\prime} \in w^{\prime}} \epsilon_{i}\left(\mathcal{I}_{i}\left(X^{\prime}\right)\right)\right) \neq \emptyset \\
\Longrightarrow & \varphi(i: R) \cap\left(\bigcap_{X \in \widehat{w}} \varphi(X) \times \bigcap_{X^{\prime} \in \widehat{w}^{\prime}} \varphi\left(X^{\prime}\right)\right) \neq \emptyset
\end{aligned}
$$

There exists a pair $\left\langle e, e^{\prime}\right\rangle$ in $\varphi(i: R) \cap\left(\bigcap_{X \in \widehat{w}} \varphi(X) \times \bigcap_{X^{\prime} \in \widehat{w}^{\prime}} \varphi\left(X^{\prime}\right)\right)$. Let us name $W$ the set $\widehat{w} \cup\{X \in \mathscr{C} \backslash \widehat{w} \mid e \in \varphi(X)\}$ and $W^{\prime}$ the set $\widehat{w^{\prime}} \cup\left\{X^{\prime} \in \mathscr{C} \backslash \widehat{w^{\prime}} \mid e^{\prime} \in \varphi\left(X^{\prime}\right)\right\}$. By definition, $\widehat{w} \subseteq$ $W, \widehat{w^{\prime}} \subseteq W^{\prime}, e \in \varphi\left(C_{W}\right)$ and $e^{\prime} \in \varphi\left(C_{W^{\prime}}\right)$. This proves that $\varphi(R) \cap\left(\varphi\left(C_{W}\right) \times \varphi\left(C_{W^{\prime}}\right)\right) \neq \emptyset$. According to the construction of $\Phi_{\Omega}$, this ensures that $\left\langle W, W^{\prime}, i: R\right\rangle \in \Omega$ which establishes the proof.

\subsubsection{Consistency of $\widehat{\mathbf{A}}_{\Omega}$.}

Construction of a model of the alignment ontology. We only update the previous construction by adding the interpretation of roles.

4. For all $R \in \mathscr{R}$ we define $\mathcal{I}_{A}(R)=\left\{\left\langle W, W^{\prime}\right\rangle \in \Omega \times \Omega \mid\left\langle W, W^{\prime}, R\right\rangle \in \Phi_{\Omega}\right\}$;

Checking satisfaction of axioms. We do not check the satisfaction of the axioms already present in the first version. So, these are the only necessary proofs.

4. Let assume there is a role correspondence $i: R \stackrel{\sqsubseteq}{\Leftrightarrow}: S \in A_{i j}$. Therefore, $i: R \sqsubseteq$ $j: S \in \widehat{\mathbf{A}}_{\Omega}$ is an axiom in $\widehat{\mathbf{A}}_{\Omega}$. Let $\left\langle W, W^{\prime}\right\rangle \in \mathcal{I}_{A}(i: R)$. By construction of $\mathcal{I}_{A}$, $\left\langle W, W^{\prime}, i: R\right\rangle \in \Phi_{\Omega}$. So by construction of $\Phi_{\Omega}, \varphi(i: S) \cap\left(\varphi\left(C_{W}\right) \times \varphi\left(C_{W^{\prime}}\right)\right) \neq \emptyset$. But

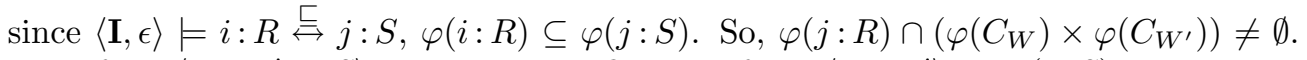
Therefore, $\left\langle W, W^{\prime}, j: S\right\rangle \in \Phi_{\Omega}$, so by definition of $\mathcal{I}_{A},\left\langle W, W^{\prime}\right\rangle \in \mathcal{I}_{A}(j: S)$.

5. If $\left\langle W, W^{\prime}, R\right\rangle \in \Phi_{\Omega}$ then $\left\{a_{W}\right\} \sqsubseteq \exists R .\left\{a_{W^{\prime}}\right\} \in \widehat{\mathbf{A}}_{\Omega}$. It was proved above that $\left\langle W, W^{\prime}, R\right\rangle \in \Phi_{\Omega}$ implies $\left\langle W, W^{\prime}\right\rangle \in \mathcal{I}_{A}(R)$ and since $\mathcal{I}_{A}\left(a_{W}\right)=W$ and $\mathcal{I}_{A}\left(a_{W^{\prime}}\right)=$ $a_{W^{\prime}}$, so $\mathcal{I}_{A}\left(a_{W}\right) \in \mathcal{I}_{A}\left(\exists R .\left\{a_{W^{\prime}}\right\}\right)$. 
6. If $\left\langle W, W^{\prime}, R\right\rangle \notin \Phi_{\Omega}$ then $\left\{a_{W}\right\} \sqsubseteq \forall R . \neg\left\{a_{W^{\prime}}\right\} \in \widehat{\mathbf{A}}_{\Omega}$. When neither $W$ nor $W^{\prime}$ is in $\Omega$, the axiom is obviously satisfied by $\mathcal{I}_{A}$. Otherwise, $\mathcal{I}_{A}\left(a_{W}\right)=W$ and $\left.\mathcal{I}_{A}\left(a_{w^{\prime}}\right)\right)=W^{\prime}$, and $\left\langle W, W^{\prime}\right\rangle \notin \mathcal{I}_{A}(R)$ by construction of $\mathcal{I}_{A}$. This clearly implies that $\mathcal{I}_{A} \models\left\{a_{W}\right\} \sqsubseteq$ $\forall R . \neg\left\{a_{W^{\prime}}\right\}$.

\subsubsection{Consistency of $\widehat{O}_{\Omega^{i}}$.}

The proof of consistency of $\widehat{O}_{\Omega^{i}}$ will necessitate two additional results. They assert the non-emptiness of sets in which $b_{w, w^{\prime}}^{R}$ and $\beta_{W, W^{\prime}}^{R}$ will be interpreted.

The next lemma will be used to define an interpretation of the individual $\beta_{W, W^{\prime}}^{R}$.

Lemma 6 If $\left\langle W, W^{\prime}, i: R\right\rangle \in \Phi_{\Omega}$ then there exists

$$
\left\langle e, e^{\prime}\right\rangle \in\left(\bigcap_{\left.X \in \mathscr{C}_{i} \backslash W\right|_{i}} \Delta_{i} \backslash \mathcal{I}_{i}(X)\right) \times\left(\bigcap_{\left.X \mathscr{C}^{\prime} \in \mathscr{C}_{i} \backslash W^{\prime}\right|_{i}} \Delta_{i} \backslash \mathcal{I}_{i}\left(X^{\prime}\right)\right)
$$

such that $\left\langle e, e^{\prime}\right\rangle \in \mathcal{I}_{i}(R)$.

Before proceeding with the proof, we have to give a meaning to the specific set $\bigcap_{X \in \emptyset} \Delta_{i} \backslash \mathcal{I}_{i}(X)$. By convention, we assume that this set is equal to $\Delta_{i}$, and we also assume that the set

$\bigcap \Delta_{\epsilon} \backslash \varphi(i: X)$ is equal to $\Delta_{\epsilon}$. $\left.X \in \mathscr{C}_{i} \backslash W\right|_{i}$

Proof: Let us assume that $\left\langle W, W^{\prime}, i: R\right\rangle \in \Phi_{\Omega}$. We can devise the following implications:

$$
\begin{aligned}
& \left\langle W, W^{\prime}, i: R\right\rangle \in \Phi_{\Omega} \\
\Longrightarrow & \varphi(i: R) \cap\left(\varphi\left(C_{W}\right) \times \varphi\left(C_{W^{\prime}}\right)\right) \neq \emptyset \\
\Longrightarrow & \varphi(i: R) \cap\left(\bigcap_{X \in \mathscr{C} \backslash W} \bar{\varphi}(X) \times \bigcap_{X^{\prime} \in \mathscr{C} \backslash W^{\prime}} \bar{\varphi}\left(X^{\prime}\right)\right) \neq \emptyset \\
\Longrightarrow & \epsilon_{i}\left(\mathcal{I}_{i}(R)\right) \cap\left(\bigcap_{\left.X \in \mathscr{C}_{i} \backslash W\right|_{i}} \Delta_{\epsilon} \backslash \varphi(i: X) \times \bigcap_{\left.X^{\prime} \in \mathscr{C}_{i} \backslash W^{\prime}\right|_{i}} \Delta_{\epsilon} \backslash \varphi\left(i: X^{\prime}\right)\right) \neq \emptyset \\
\Longrightarrow & \mathcal{I}_{i}(R) \cap\left(\bigcap_{\left.X \in \mathscr{C}_{i} \backslash W\right|_{i}} \Delta_{i} \backslash \mathcal{I}_{i}(X) \times \bigcap_{\left.X^{\prime} \in \mathscr{C}_{i} \backslash W^{\prime}\right|_{i}} \Delta_{i} \backslash \mathcal{I}_{i}\left(X^{\prime}\right)\right) \neq \emptyset
\end{aligned}
$$

This last lemma will be used to define an interpretation of the individual $b_{w, w^{\prime}}^{R}$.

Lemma 7 If $\left\langle w, w^{\prime}, R\right\rangle \in \Phi_{\Omega^{i}}$ then $\mathcal{I}_{i}\left(C_{w}^{\mathscr{C}_{i}}\right) \cap \mathcal{I}_{i}\left(\exists R . C_{w^{\prime}}^{\mathscr{C}_{i}}\right) \neq \emptyset$. 
Proof: By contruction of $\Phi_{\Omega^{i}}$,

$$
\begin{aligned}
& \left\langle w, w^{\prime}, R\right\rangle \in \Phi_{\Omega^{i}} \\
\Longrightarrow & \mathcal{I}_{i}(R) \cap\left(\mathcal{I}_{i}\left(C_{w}^{\mathscr{C}_{i}}\right) \times \mathcal{I}_{i}\left(C_{w^{\prime}}^{\mathscr{C}_{i}}\right)\right) \neq \emptyset \\
\Longrightarrow & \mathcal{I}_{i}\left(C_{w}^{\mathscr{C}_{i}}\right) \cap \mathcal{I}_{i}\left(\exists R \cdot C_{w^{\prime}}^{\mathscr{C}_{i}}\right) \neq \emptyset
\end{aligned}
$$

Construction of a model of the extended ontology. We build the model of $\widehat{O}_{\Omega^{i}}$ by extending the construction already started in Sect. 6.2.3.

8. for all $b_{w, w^{\prime}}^{R}$ added by Def. 19 to $\widehat{O}_{\Omega^{i}}$ with assertion $\left(C_{w}^{\mathscr{C}_{i}} \sqcap \exists R . C_{w^{\prime}}^{\mathscr{C}_{i}}\right)\left(b_{w, w^{\prime}}^{R}\right)$, we define $\mathcal{I}_{i}^{\prime}\left(b_{w, w^{\prime}}^{R}\right)=x$ for any $x \in \mathcal{I}_{i}\left(C_{w}^{\mathscr{C}_{i}}\right) \cap \mathcal{I}_{i}\left(\exists R . C_{w^{\prime}}^{\mathscr{C}_{i}}\right)$ (which exists, according to Lem. 7);

9. for all $C_{W, W^{\prime}}^{R}$ added by Def. 19 to $\widehat{O}_{\Omega^{i}}$, we define $\mathcal{I}_{i}^{\prime}\left(C_{W, W^{\prime}}^{R}\right)$ as the set

$$
\left\{e \in \bigcap_{\left.X \in \mathscr{C}_{i} \backslash W\right|_{i}} \Delta_{i} \backslash \mathcal{I}_{i}(X) \mid \exists e^{\prime} \in \bigcap_{\left.X^{\prime} \in \mathscr{C}_{i} \backslash W^{\prime}\right|_{i}} \Delta_{i} \backslash \mathcal{I}_{i}\left(X^{\prime}\right) \text { s.t. }\left\langle e, e^{\prime}\right\rangle \in \mathcal{I}_{i}(R)\right\}
$$

10. for all $\beta_{W, W^{\prime}}^{R}$ added by Def. 19 to $\widehat{O}_{\Omega^{i}}$ with assertion $C_{W, W^{\prime}}^{R}\left(\beta_{W, W^{\prime}}^{R}\right)$, we define $\mathcal{I}_{i}^{\prime}\left(\beta_{W, W^{\prime}}^{R}\right)=$ $x$ for any $x \in \mathcal{I}_{i}^{\prime}\left(C_{W, W^{\prime}}^{R}\right)$ (which exists according to Lem. 6 .

Checking satisfaction of axioms. As for the consistency of the alignment ontology, we only present the proofs of satisfaction of the new axioms.

7. Satisfaction of $\left(C_{w}^{\mathscr{C}_{i}} \sqcap \exists R . C_{w^{\prime}}^{\mathscr{C}_{i}}\right)\left(b_{w}\right)$. Let $\left\langle w, w^{\prime}, R\right\rangle \in \Phi_{\Omega^{i}}$. By Lem. 7 and the construction of $\mathcal{I}_{i}^{\prime}$, we have $\mathcal{I}_{i}^{\prime}\left(b_{w}\right) \in \mathcal{I}_{i}^{\prime}\left(C_{w}^{\mathscr{C}_{i}} \sqcap \exists R . C_{w^{\prime}}^{\mathscr{C}_{i}}\right)$.

8. Satisfaction of $C_{w}^{\mathscr{C}_{i}} \sqsubseteq \forall R$. $\neg C_{w^{\prime}}^{\mathscr{C}_{i}}$. Let $\left\langle w, w^{\prime}, R\right\rangle \notin \Phi_{\Omega^{i}}$. This is a direct consequence of the construction of $\Phi_{\Omega^{i}}$.

9. Let $W, W^{\prime} \in \Omega$ and $R \in \mathscr{R}_{i}$.

(a) Satisfaction of $C_{W, W^{\prime}}^{R} \sqsubseteq C_{W}^{\top}$. By construction of $\mathcal{I}_{i}^{\prime}$,

$$
\mathcal{I}_{i}^{\prime}\left(C_{W, W^{\prime}}^{R}\right) \subseteq \bigcap_{X \in \mathscr{C}_{i} \backslash w}\left(\Delta_{i} \backslash \mathcal{I}_{i}(X)\right)=\mathcal{I}_{i}^{\prime}\left(C_{W}^{\top}\right)
$$

(b) Satisfaction of $C_{W, W^{\prime}}^{R} \sqsubseteq \exists R . C_{W^{\prime}, W}^{R^{-}}$and $C_{W, W^{\prime}}^{R}\left(\beta_{W, W^{\prime}}^{R}\right)$. Assume that $\left\langle W, W^{\prime}, i\right.$ : $R\rangle \in \Phi_{\Omega}$. Let $e \in \mathcal{I}_{i}^{\prime}\left(C_{W, W^{\prime}}^{R}\right)$. The construction of $\mathcal{I}_{i}^{\prime}$ implies that there exists $e^{\prime} \in \bigcap_{\left.X^{\prime} \in \mathscr{C}_{i} \backslash W^{\prime}\right|_{i}} \Delta_{i} \backslash \mathcal{I}_{i}\left(X^{\prime}\right)$ such that $\left\langle e, e^{\prime}\right\rangle \in \mathcal{I}_{i}(R)$. But therefore, $e^{\prime}$ is such that 
there exists $e \in \bigcap_{\left.X \in \mathscr{C}_{i} \backslash W\right|_{i}} \Delta_{i} \backslash \mathcal{I}_{i}(X)$ with $\left\langle e, e^{\prime}\right\rangle \in \mathcal{I}_{i}^{\prime}\left(R^{-}\right)$. So $e^{\prime} \in \mathcal{I}_{i}^{\prime}\left(C_{W^{\prime}, W}^{R^{-}}\right)$. Therefore $e \in \mathcal{I}^{\prime}\left(\exists R . C_{W^{\prime}, W}^{R^{-}}\right)$. More generally, $\mathcal{I}_{i}^{\prime}\left(C_{W, W^{\prime}}^{R}\right) \subseteq \mathcal{I}_{i}^{\prime}\left(\exists R . C_{W^{\prime}, W}^{R^{-}}\right)$. The satisfaction of $C_{W, W^{\prime}}^{R}\left(\beta_{W, W^{\prime}}^{R}\right)$ is a direct consequence of Lem. 6 and the construction of $\mathcal{I}_{i}^{\prime}$.

(c) Satisfaction of $C_{W, W^{\prime}}^{R} \sqsubseteq \forall R . \neg C_{W, W^{\prime}}^{R^{-}}$. Let us assume that $\left\langle W, W^{\prime}, i: R\right\rangle \notin \Phi_{\Omega}$. So $\varphi(R) \cap\left(\varphi\left(C_{W}\right) \times \varphi\left(C_{W^{\prime}}\right)\right)=\emptyset$. Let us consider $\left\langle e, e^{\prime}\right\rangle \in \mathcal{I}_{i}^{\prime}(R)$ such that $e \in \mathcal{I}_{i}^{\prime}\left(C_{W, W^{\prime}}^{R}\right)$ and $e^{\prime} \in \mathcal{I}_{i}^{\prime}\left(C_{W^{\prime}, W}^{R^{-}}\right)$. Since $\mathcal{I}_{i}^{\prime} \models C_{W, W^{\prime}}^{R} \sqsubseteq C_{W}^{\top}$ (proved above), $e \in$ $\mathcal{I}_{i}^{\prime}\left(C_{W}^{\top}\right)$ and $\mathcal{I}_{i}^{\prime} \models C_{W^{\prime}, W}^{R^{-}} \sqsubseteq C_{W^{\prime}}^{\top}$, implies that $e^{\prime} \in \mathcal{I}_{i}^{\prime}\left(C_{W^{\prime}}^{\top}\right)$. So, by construction of $\mathcal{I}_{i}^{\prime},\left\langle\epsilon_{i}(e), \epsilon_{i}\left(e^{\prime}\right)\right\rangle \in \varphi(i: R) \cap\left(\varphi\left(C_{W}\right) \times \varphi\left(C_{W^{\prime}}\right)\right)$. This contradict the initial assumption. Therefore, there is no such $e$ and $e^{\prime}$.

10. Satisfaction of $\exists R . \top \sqsubseteq \bigsqcup_{W, W^{\prime} \in \Omega} C_{W, W^{\prime}}^{R}$. Let $e \in \mathcal{I}_{i}^{\prime}(\exists R . \top)$, which means that there exists $e^{\prime} \in \Delta_{i}$ such that $\left\langle e, e^{\prime}\right\rangle \in \mathcal{I}_{i}^{\prime}(R)$. Using the function $\Theta$ defined in previous section $\left(\Theta: \Delta_{i} \rightarrow 2^{\mathscr{C}}\right.$ such that for all $e \in \Delta_{i}, \Theta(e)=\left\{X \in \mathscr{C}_{i} \mid e \in \mathcal{I}_{i}(X)\right)$, we have $e \in \mathcal{I}_{i}\left(C_{\Theta(e)}^{\mathscr{C}_{i}}\right)$ and $e^{\prime} \in \mathcal{I}_{i}\left(C_{\Theta\left(e^{\prime}\right)}^{\mathscr{C}_{i}}\right)$. So $\left\langle e, e^{\prime}\right\rangle \in \mathcal{I}_{i}(R) \cap\left(\mathcal{I}_{i}\left(C_{w}^{\mathscr{C}_{i}}\right) \times \mathcal{I}_{i}\left(C_{w^{\prime}}^{\mathscr{C}_{i}}\right)\right)$. This implies that $\left\langle\Theta(e), \Theta\left(e^{\prime}\right), R\right\rangle \Phi_{\Omega^{i}}$. By definition of $\Phi_{\Omega^{i}}$, there exists $W, W^{\prime} \in \Omega$ such that $\left.\Theta(e) \subseteq W\right|_{i}$ and $\left.\Theta\left(e^{\prime}\right) \subseteq W^{\prime}\right|_{i}$ and $\left\langle W, W^{\prime}, i: R\right\rangle \in \Phi_{\Omega}$. By construction of $\mathcal{I}_{i}^{\prime}$ and what precedes, we deduce that $e \in \mathcal{I}_{i}^{\prime}\left(C_{W, W^{\prime}}^{R}\right)$. So finally, $\mathcal{I}^{\prime} \models \exists R$. $\top \sqsubseteq \bigsqcup_{W, W^{\prime} \in \Omega} C_{W, W^{\prime}}^{R}$.

\section{Conclusion and Future Work}

We have introduced a new formalism for distributed reasoning with aligned descriptions logics. Although we do not pretend to replace or improve existing formalisms, we believe it has several advantages. In terms of paradigm, the differentiation of local and global reasoning is, in our opinion, more appropriate to the case of independently produced ontologies and ontology alignments. The reasoning task is not focused on one particular local knowledge. It is rather adapted to deducing knowledge about the overall system. In particular, it can be use to compose alignments. As a matter of technical quality, it has the advantage of being decidable iff local languages are decidable. Moreover, our reasoning procedure strictly encapsulate local reasoning so that it is never expected that the content of a local ontology is actually accessed by the global reasoner. As a result, we would advertise this formalism as an appropriate modular ontology language in the Semantic Web. Additionally, some inferences do not transfer from local to global level. Consequently, potential inconsistencies are semantically avoided.

Nevertheless, there are still important drawbacks that need to be filled. The most important one is the algorithmic complexity of reasoning, which is far from reasonable with 
the naive procedure we proposed. A real life implementation would inevitably require optimization. Additionally, we have not yet investigated reasoning with concrete domains. Moreover, we do not allow concept-role correspondences, although these are well formalized in DDL [12].

As far as future research is concerned, we envisage the following directions:

- implement a slightly optimized version of the algorithm;

- optimize and test it on real life examples;

- extend the theorem to description logics with concrete domains;

- integrate the reasoner our modular ontology framework [11;

- define the notion of local reasoning with respect to to a distributed system;

- enrich the alignment language with constructors of its own. [10.

The last item is important to reason with an expressive alignment language as found in

\section{References}

[1] Baader, F., Lutz, C., and Suntisrivaraporn, B. CEL-A Polynomial-time Reasoner for Life Science Ontologies. In Automated Reasoning, Third International Joint Conference, IJCAR 2006, Seattle, WA, USA, August 17-20, 2006, Proceedings (2006), U. Furbach and N. Shankar, Eds., vol. 4130 of Lecture Notes in Computer Science, Springer-Verlag GmbH, pp. 287-291.

[2] Bao, J., Caragea, D., and Honavar, V. G. A distributed Tableau Algorithm for Package-based Description Logics. In Proceedings of the ECAI Workshop on on Context Representation and Reasoning (2006), C. Ghidini, L. Serafini, and P. Bouquet, Eds.

[3] Bao, J., Caragea, D., and Honavar, V. G. On the Semantics of Linking and Importing in Modular Ontologies. In The Semantic Web - ISWC 2006, 5th International Semantic Web Conference, ISWC 2006, Athens, GA, USA, November 5-9, 2006, Proceedings (Nov. 2006), I. F. Cruz, S. Decker, D. Allemang, C. Preist, D. Schwabe, P. Mika, M. Uschold, and L. Aroyo, Eds., vol. 4273 of Lecture Notes in Computer Science, Springer-Verlag GmbH, pp. 72-86.

[4] Borgida, A., and Serafini, L. Distributed description logics: Assimilating information from peer sources. Journal on Data Semantics 1 (2003), 153-184.

[5] Bouquet, P., Giunchiglia, F., van Harmelen, F., Serafini, L., and StuckEnschmidt, H. C-OWL: Contextualizing ontologies. In Proc. Second International Semantic Web Conference (ISWC'03) (Sanibel Island, FL, USA, Oct. 20 2003), and 
Katia P. Sycara and J. Mylopoulos, Eds., vol. 2870 of Lecture Notes in Computer Science, Springer-Verlag GmbH.

[6] Cuenca-Grau, B., Horrocks, I., Kazakov, Y., and Sattler, U. A logical framework for modularity of ontologies. In Proc. of 20th International Joint Conference on Artificial Intelligence (IJCAI'07) (2007), M. M. Veloso, Ed., pp. 298-303.

[7] Cuenca-Grau, B., And Kutz, O. Modular Ontology Languages Revisisted. In SWeCKa 2007: Proc. of the IJCAI-2007 Workshop on Semantic Web for Collaborative Knowledge Acquisition, Hyderabad, India, January 7, 2007 (2007), V. G. Honavar, , D. Caragea, D. Mladenic, and Y. Sure, Eds.

[8] Cuenca-Grau, B., Parsia, B., and Sirin, E. Combining OWL ontologies using $\mathcal{E}$-Connections. Journal of Web Semantics 4, 1 (2006), 40-59.

[9] Euzenat, J. Semantic precision and recall for ontology alignment evaluation. In Proc. 20th International Joint Conference on Artificial Intelligence (IJCAI), Hyderabad (IN) (Menlo Park (CA US), 2007), AAAI Press, pp. 248-253.

[10] Euzenat, J., Scharffe, F., And Zimmermann, A. Expressive alignment language and implementation. deliverable 2.2.10, Knowledge Web NoE, 2007.

[11] Euzenat, J., Zimmermann, A., And Freitas, F. Alignment-based modules for encapsulating ontologies. In Second International Workshop on Modular Ontologies (WOMO 2007) (June 2007), B. Cuenca-Grau, V. G. Honavar, A. Schlicht, and F. Wolter, Eds., pp. 32-45.

[12] Ghidini, C., And Serafini, L. On relating heterogeneous elements from different ontologies. In Proceedings of the Sixth International and Interdisciplinary Conference on Modeling and Using Context (CONTEXT'07) (2007), Springer-Verlag GmbH.

[13] McGuinness, D. L., And van Harmelen, F. OWL Web Ontology Language Overview W3C Recommendation 10 February 2004. Tech. rep., World Wide Web Consortium (W3C), Feb. 102004.

[14] Meilicke, C., Stuckenschmidt, H., And Tamilin, A. Improving automatically created mappings using logical reasoning. In Proceedings of the Worshop on Ontology Matching (2006), R. Benjamin, J. Euzenat, N. F. Noy, P. Shvaiko, H. Stuckenschmidt, and M. Uschold, Eds.

[15] Meilicke, C., Stuckenschmidt, H., And Tamilin, A. Repairing ontology mappings. In Proceedings of the Twenty-Second AAAI Conference on Artificial Intelligence, July 22-26, 2007, Vancouver, British Columbia, Canada (2007), AAAI Press, pp. 14081413. 
[16] Patel-Schneider, P. F. System description: Dlp. In 17th International Conference on Automated Deduction, CADE 2000 (2007), D. A. McAllester, Ed., vol. 1831, Springer-Verlag GmbH, pp. 297-301.

[17] Racer Systems GmbH \& Co. KG. RacerPro Reference Manual, 2007.

[18] Serafini, L., And Tamilin, A. DRAGO: Distributed Reasoning Architecture for the Semantic Web. In The Semantic Web: Research and Applications, Second European Semantic Web Conference, ESWC 2005, Heraklion, Crete, Greece, May 29 - June 1, 2005, Proceedings (2005), A. Gomez-Perez and J. Euzenat, Eds., vol. 3532 of Lecture Notes in Computer Science, Springer-Verlag GmbH, pp. 361-376.

[19] Sirin, E., Parsia, B., Cuenca-Grau, B., Kalyanpur, A., and Katz, Y. Pellet: a pratical OWL-DL reasoner. Journal of Web Semantics 5, 2 (2007), 51-53.

[20] Tsarkov, D., and Horrocks, I. FaCT++ Description Logic Reasoner: System Description. In Automated Reasoning, Third International Joint Conference, IJCAR 2006, Seattle, WA, USA, August 17-20, 2006, Proceedings (2006), U. Furbach and N. Shankar, Eds., vol. 4130 of Lecture Notes in Computer Science, Springer-Verlag GmbH, pp. 292-297.

[21] Zimmermann, A. Integrated Distributed Description Logics. In Proceedings of the 20th International Workshop on Description Logics DL'07 (June 2007), D. Calvanese, E. Franconi, V. Haarslev, D. Lembo, B. Motik, S. Tessaris, and A.-Y. Turhan, Eds., Bolzano University Press, pp. 507-514.

[22] Zimmermann, A., And Euzenat, J. Three Semantics for Distributed Systems and their Relations with Alignment Composition. In The Semantic Web - ISWC 2006, 5th International Semantic Web Conference, ISWC 2006, Athens, GA, USA, November 5-9, 2006, Proceedings (Nov. 2006), I. F. Cruz, S. Decker, D. Allemang, C. Preist, D. Schwabe, P. Mika, M. Uschold, and L. Aroyo, Eds., vol. 4273 of Lecture Notes in Computer Science, Springer-Verlag GmbH, pp. 16-29. 
Unité de recherche INRIA Rhône-Alpes 655, avenue de l'Europe - 38334 Montbonnot Saint-Ismier (France)

Unité de recherche INRIA Futurs : Parc Club Orsay Université - ZAC des Vignes 4, rue Jacques Monod - 91893 ORSAY Cedex (France)

Unité de recherche INRIA Lorraine : LORIA, Technopôle de Nancy-Brabois - Campus scientifique 615, rue du Jardin Botanique - BP 101 - 54602 Villers-lès-Nancy Cedex (France)

Unité de recherche INRIA Rennes : IRISA, Campus universitaire de Beaulieu - 35042 Rennes Cedex (France)

Unité de recherche INRIA Rocquencourt : Domaine de Voluceau - Rocquencourt - BP 105 - 78153 Le Chesnay Cedex (France)

Unité de recherche INRIA Sophia Antipolis : 2004, route des Lucioles - BP 93 - 06902 Sophia Antipolis Cedex (France) 